\title{
23. OXYGEN AND CARBON ISOTOPE STRATIGRAPHY OF ODP HOLE 653A AND SITE 654: THE PLIOCENE-PLEISTOCENE GLACIAL HISTORY RECORDED IN THE TYRRHENIAN BASIN (WEST MEDITERRANEAN) ${ }^{1}$
}

\author{
Colette Vergnaud Grazzini, ${ }^{2}$ Jean François Saliège, ${ }^{2}$ Marie José Urrutiaguer, ${ }^{2}$ and Alessandro Iannace ${ }^{2}$
}

\begin{abstract}
Stable isotope analysis of two species (or groups of species) of planktonic foraminifers: Globigerinoides ruber (or $G$. obliquus and G. obliquus extremus) and Globigerina bulloides (or G. falconensis and G. obesa) from ODP Hole 653A and Site 654 in the Tyrrhenian basin, records the Pliocene-Pleistocene glacial history of the Northern Hemisphere. The overall increase in mean $\delta^{18} \mathrm{O}$ values through the interval $4.6-0.08 \mathrm{Ma}$ is $1.7 \%$ for $G$. bulloides and $1.5 \%$ for $G$. ruber. The time interval 3.1-2.5 Ma corresponds to an important phase of ${ }^{18} \mathrm{O}$ enrichment for planktonic foraminifers. In this interval, glacial $\delta^{18} \mathrm{O}$ values of both species $G$. bulloides and $G$. ruber increase by about $1 \%$, this increase being more progressive for $G$. ruber than for $G$. bulloides. The increase of interglacial $\delta^{18} \mathrm{O}$ values is higher for $G$. bulloides $(1.5 \%)$ than for the G.ruber group (1\%). These data suggest a more pronounced seasonal stratification of the water masses during interglacial phases. Large positive $\delta^{18} \mathrm{O}$ fluctuations of increasing magnitude are also recorded at 2.25 and 2.15 Ma by $G$. bulloides and appear to be diachronous with those of Site 606 in the Atlantic Ocean. Other events of increasing $\delta^{18} \mathrm{O}$ values are recorded between 1.55 and $1.3 \mathrm{Ma}$, at $0.9 \mathrm{Ma}, 0.8 \mathrm{Ma}$, and near $0.34 \mathrm{Ma}$. In the early Pliocene the $\delta^{18} \mathrm{O}$ variability recorded by the planktonic species $G$. bulloides was higher in the Mediterranean than in the Atlantic at the same latitude. This suggests that important cyclic variations in the water budget of the Mediterranean occurred since that time. Step increases in the $\delta^{18} \mathrm{O}$ variability are synchronous with those of the open ocean at 0.9 and $0.34 \mathrm{Ma}$. The higher variability as well as the higher amplitude of the peaks of ${ }^{18} \mathrm{O}$ enrichment may be partly accounted for by increase of dryness over the Mediterranean area. In particular the high amplitude $\delta^{18} \mathrm{O}$ fluctuations recorded between 3.1 and $2.1 \mathrm{Ma}$ are correlated with the onset of a marked seasonal contrast and a summer dryness, revealed by pollen analyses. Strong fluctuations towards $\delta^{13} \mathrm{C}$ values higher than modern ones are recorded by the $G$. ruber group species before $1.7 \mathrm{Ma}$ and suggest a high production of phytoplankton. When such episodes of high primary production are correlated with episodes of decreasing ${ }^{13} \mathrm{C}$ content of $\mathrm{G}$. bulloides, they are interpreted as the consequence of a higher stratification of the upper water masses resulting itself from a marked seasonality. Such episodes occur between 4.6 and 4.05 Ma, 3.9 and 3.6 Ma, and 3.25 and 2.66 Ma. The interval 2.66-1.65 Ma corresponds to a weakening of the stratification of the upper water layers. This may be related to episodes of cooling and increasing dryness induced by the Northern Hemisphere Glaciations. The Pleistocene may have been a less productive period. The transition from highly productive to less productive surface waters also coincides with a new step increase in dryness and cooling, between 1.5 and $1.3 \mathrm{Ma}$. The comparison of the ${ }^{13} \mathrm{C}$ records of $G$. ruber and $G$. bulloides in fact suggests that a high vertical convection became a dominant feature after $2.6 \mathrm{Ma}$. Increases in the nutrient input and the stratification of the upper water masses may be suspected, however, during short episodes near $0.86 \mathrm{Ma}$ (isotopic stage 25), $0.57-0.59 \mathrm{Ma}$ (isotopic stage 16 ), $0.49 \mathrm{Ma}$ (isotopic stage 13), 0.4-0.43 Ma (isotopic stage 11), and 0.22 and $0.26 \mathrm{Ma}$ (part of isotopic stage 7 and transition 7/8). In fact, changes in the $\mathrm{CO}_{2}$ balance within the different water masses of the Tyrrhenian basin as well as in the local primary production did not follow the general patterns of the open ocean.
\end{abstract}

\section{INTRODUCTION}

A steadily growing database of long term oxygen isotopic records is being generated for Mediterranean Pliocene-Pleistocene deep sea drilled sequences (DSDP Site 125 in the Ionian sea and Site 132 in the Tyrrhenian sea: Thunell and Williams, 1983; Keigwin and Thunell, 1979) and land-based marine sequences from southern Italy (the Capo-Rossello composite section: Thunell et al., 1985; Gudjonsson and Van der Zwaan, 1985; Van der Zwaan and Gudjonsson, 1986; the Monte Signa section: Gudjonsson, 1987; and the Semaforo-Vrica composite section: Comb ourieu Nebout, 1987). From the $\delta^{18} \mathrm{O}$ record at the DSDP sites, stepwise changes in the paleoclimate of the Mediterranean have been inferred (Thunell and Williams, 1983); these steps appeared to be synchronous with the climatic steps recognized in the Pliocene-Pleistocene isotopic records of the Atlantic and $\mathrm{Pa}$ cific Oceans (Shackleton and Opdyke, 1976, 1977; Shackleton and

\footnotetext{
${ }^{1}$ Kastens, K. A., Mascle, J., et al., 1990. Proc. ODP, Sci. Results, 107: College Station, TX (Ocean Drilling Program).

2 Laboratoire de Géologie Dynamique, 4 Place Jussieu, 75252 Paris Cédex 05, France.
}

Cita, 1979; Prell, 1982; Shackleton and Hall, 1983; Leonard et al., 1983; Vergnaud Grazzini et al., 1983). More recently, high resolution studies and spectral analyses of the oxygen isotope signal tend to prove, however, that changes in the climate regime that occurred either in the middle Pleistocene or in the late Pliocene might have been complicated responses with a certain duration to orbital forcing, rather than abrupt shifts from one regime to another (Fillon and Williams, 1983; Hodell et al., 1985; Imbrie et al., 1984; Keigwin, 1987; Loubere, 1988; Martinson et al., 1987; Ruddiman et al., 1986; Shackleton and Hall, 1984; Shackleton et al., 1984; Williams et al., 1988, Zimmermann et al., 1985; among others).

ODP Leg 107, in the Tyrrhenian basin, provided the opportunity to obtain, through HPC coring, continuous deep sea sediment records, dating beds to at least the opening of the basin. ODP Site 653 was cored with the primary objective of collecting a Pliocene-Pleistocene sequence that would serve as a "deep sea Mediterranean type section" for both stratigraphic and paleoceanographic studies. In this paper we present Pliocene-Pleistocene oxygen and carbon isotope records for ODP Hole 653A as well as for Site 654 .

Because both the core recovery and the average sampling interval (from 6 k.y. to 10 k.y. for the Pleistocene section and 
around $12 \mathrm{k} . \mathrm{y}$. for the Pliocene one at Hole 653A were higher than at other sites (allowing a reasonable time resolution for the last $4.6 \mathrm{Ma}$ ), we have used the isotopic record and the faunal events determined at Hole $653 \mathrm{~A}$ to calibrate the other sites. At Site 654 , core recovery was only slightly greater than $50 \%$. As a consequence of this poor recovery the stable isotope records at this site are unsuitable for detailed stratigraphic studies. Even the record at Hole $653 \mathrm{~A}$ is less than ideal because of active volcanism and tectonism in the Tyrrhenian basin during the period under study. A preliminary sedimentological survey carried out by Glaçon (pers. comm., 1988) reveals numerous disturbed levels, either by tuffitic residues or by small intercalations of turbidites with varying thickness at all sites.

Despite these disadvantages, we used the isotope records of ODP Hole $653 \mathrm{~A}$ and Site 654 to decipher the paleoceanographic evolution of the Mediterranean for the past 4.6 Ma and to look at the response of this semienclosed basin to the Pliocene-Pleistocene glacial history of the Northern Hemisphere. These records also should allow us to improve the Pliocene stratigraphic framework of the Mediterranean and to explore the relationships between faunal events and stable isotope events. There have been numerous attempts to demonstrate the isochroneity and/or the heterochroneity of various Neogene microfossils data between the Mediterranean and the open ocean. While the open ocean biostratigraphic data from piston cores and deep sea drills have been accurately tied to the magnetic polarity time scale, similar attempts on DSDP (Leg 42A) materials from the Mediterranean have been less successful because of drilling disturbance, reworking of sediments, and the occurrence of frequent turbidite layers. Studies on Mediterranean land base marine sequences (Rio et al.,1984) and on the present ODP Leg 107 Sites (Rio et al., this volume; Channell et al. this volume), however, have permitted more precise dating of microfossil events within the Mediterranean and emphasis of the fact that little or no diachronism exists between the Mediterranean and open ocean nannofossil data. Such data have therefore been used to establish a time scale for isotopic events older than $0.9 \mathrm{Ma}$ at Hole $653 \mathrm{~A}$ and at Site 654. This allowed in turn to compare the timing of the Pliocene-early Pleistocene stable isotope changes within the Mediterranean with the global climatic evolution.

\section{METHODS}

Coordinates, depths, and total length cored at the four studied sites are reported in Table 1 (Fig. 1). Isotopic analyses were performed on the same levels as those used by Glaçon and Vergnaud Grazzini (this volume) for biostratigraphic purposes. Some additional samples were provided by de Visser and Chamley (one sample per section) for Hole $653 \mathrm{~A}$, resulting in four samples per core. Foraminifers were picked in the fraction above $150 \mu \mathrm{m}$. Because benthic foraminifers were rare (and are still rare below $1300 \mathrm{~m}$ in the Mediterranean today), we decided to analyse two planktonic species which nowadays inhabit two different water masses: Globigerina bulloides which occurs in maximal abundances in the late spring at the depth of the thermo-halocline and Globigerinoides ruber, a species which lives in surficial waters at the end of summer and in autumn.

Table 1. Coordinates, water depths, and lengths of the Pliocene-Pleistocene sequences (with percentage of core recovery) at ODP Holes $653 \mathrm{~A}$ and $654 \mathrm{~A}$.

\begin{tabular}{ccccc}
\hline Location & Coordinates & $\begin{array}{c}\text { Water } \\
\text { depth } \\
(\mathrm{m})\end{array}$ & $\begin{array}{c}\text { Total length of the } \\
\text { Pliocene-Pleistocene } \\
\text { record }(\mathrm{m})\end{array}$ & $\begin{array}{c}\text { Core } \\
\text { recovery } \\
(\%)\end{array}$ \\
\hline Hole 653A & $\begin{array}{c}40^{\circ} 15.86^{\prime} \mathrm{N}, \\
11^{\circ} 26.99^{\prime} \mathrm{E}\end{array}$ & $2817-2828$ & 216 & 87.8 \\
Hole 654A & $\begin{array}{c}40^{\circ} 34.76^{\prime} \mathrm{N}, \\
10^{\circ} 41.80^{\prime} \mathrm{E}\end{array}$ & $2208-2217$ & 242.8 & 50.6 \\
\hline
\end{tabular}

Downcore (in the Pliocene sections), G. ruber was preceded by $G$. obliquus or $G$. obliquus extremus. A similar habitat was inferred for all these species. The deviation from isotopic equilibrium was assumed to be the same as for the modern $G$. ruber. At some levels, $G$. bulloides was replaced by $G$. falconensis, $G$. obesa, or $G$. calida; the ecological requirements of these related species is however not very well known. Paired analyses of $G$. bulloides and $G$. falconensis carried out at various levels of Site 654 , indicate a difference of $+0.30 \pm 0.1$ for $\delta^{18} \mathrm{O}$ and $-0.33 \pm 0.1$ for $\delta^{13} \mathrm{C}$. Oxygen and carbon isotopic compositions of $G$. falconensis were adjusted to $G$. bulloides to reconstruct the paleoclimatic curve (Table 2).

Standard techniques for oxygen and carbon isotopic analysis were used. Approximately 25-30 individuals of each species were picked from each sample and cleaned in an ultrasonic bath to remove fine fraction contamination. All samples were roasted under vacuum at $350^{\circ} \mathrm{C}$ for 1 hr. $\mathrm{H}_{2} \mathrm{O}$ and $\mathrm{CO}_{2}$ were extracted from the carbonate reaction with $100 \%$ orthophosphoric acid at $50^{\circ} \mathrm{C}$ and separated by a series of two freezing transfer steps. The $\mathrm{CO}_{2}$ was analyzed in an on-line VG Isogas Sira 9 triple collector mass spectrometer. All data are referred to the PDB by the standard $\delta$ notation. Overall analytical uncertainty during the time these measurements were made (based on duplicate analyses of the standard carbonate run on separate days) was $\pm 0.08 \%$ for $\delta^{18} \mathrm{O}$ and $\pm 0.03 \%_{0}$ for $\delta^{13} \mathrm{C}$ (both $1 \sigma$ values). All analytical values are given in Table 3 and Table 4 .

\section{OXYGEN ISOTOPE STRATIGRAPHY AT HOLE 653A}

The oxygen isotope records of $G$. bulloides and $G$. ruber at Hole $653 \mathrm{~A}$ are shown as a function of depth in Figure 2 and as a function of age in Figure 5. The time scale used is based on ages of major isotopic peaks as presented bv Martinson et al. (1987) down to isotopic stage 9 (around 300 k.y.), and SPECMAP ages (Imbrie et al., 1984) for stage boundaries from stage boundary $9 / 10$ down to stage boundary $19 / 20$ (744 k.y.). Below stage 22 the sampling resolution does not permit the unambiguous identification of all isotopic stages. The numbering which is proposed is therefore only tentative and may differ either from that of Ruddimann et al. (1986) or from that of Williams et al. (1988). Dates extrapolated from biostratigraphic data, following the results of Rio et al. (this volume) were used from $\sim 50 \mathrm{mbsf}$ down to the base of the record at $211 \mathrm{mbsf}$. The control points are listed in Table 5; ages have been estimated by linear interpolation between these points. Sedimentary disturbances particularly with "highly deformed" or "soupy" sediments, occur frequently and are a source of uncertainty. They are more abundant in the Pleistocene sections within Core 2 (all sections), Core 4 (Sections 1, 3, 4, 5 to 7), and Core 6 (Sections 1, 4, 5, 6).

\section{THE PLEISTOCENE (0-84.4 mbsf, 0-1.66 Ma)}

In the upper part of the record (Fig. 3), the well established late Pleistocene isotopic stages (Emiliani, 1966; Shackleton and Opdyke, 1976) can for the most part be easily recognized down to isotopic stage 23 , on the basis of the morphology of isotopic peaks (e.g., the rather well pronounced isotopic cold stages 16 (near 42 mbsf; level 6-1, $30 \mathrm{~cm}$ ) and 22 (near 53 mbsf; level 7-2, $30 \mathrm{~cm}$ ). Some stages, however, may reveal an atypical morphology (when compared to the open ocean records) because of disturbance problems.

\section{The Upper Pleistocene (0-33.5 mbsf, 0-0.45 Ma)}

The upper part of the record (from approximately 0 to 7 mbsf) was compared with the high resolution record of a piston core from the same area (Core KET $8022 ; 40^{\circ} 35.0^{\prime} \mathrm{N}, 11^{\circ} 42.5^{\prime} \mathrm{E}$, 2430 mbsf, length $8.5 \mathrm{~m}$; Paterne et al., 1986) which was studied through tephrochronology and oxygen isotope stratigraphy based on analysis of $G$. bulloides. $\delta^{18} \mathrm{O}$ values recorded by a given species at a same date and in a same geographical area are expected to be nearly identical. Making this assumption, we tried to identify the more recent isotopic substages at Hole 653A through comparison with Core KET 8022 (Table 6). It appears (Fig. 3A) 


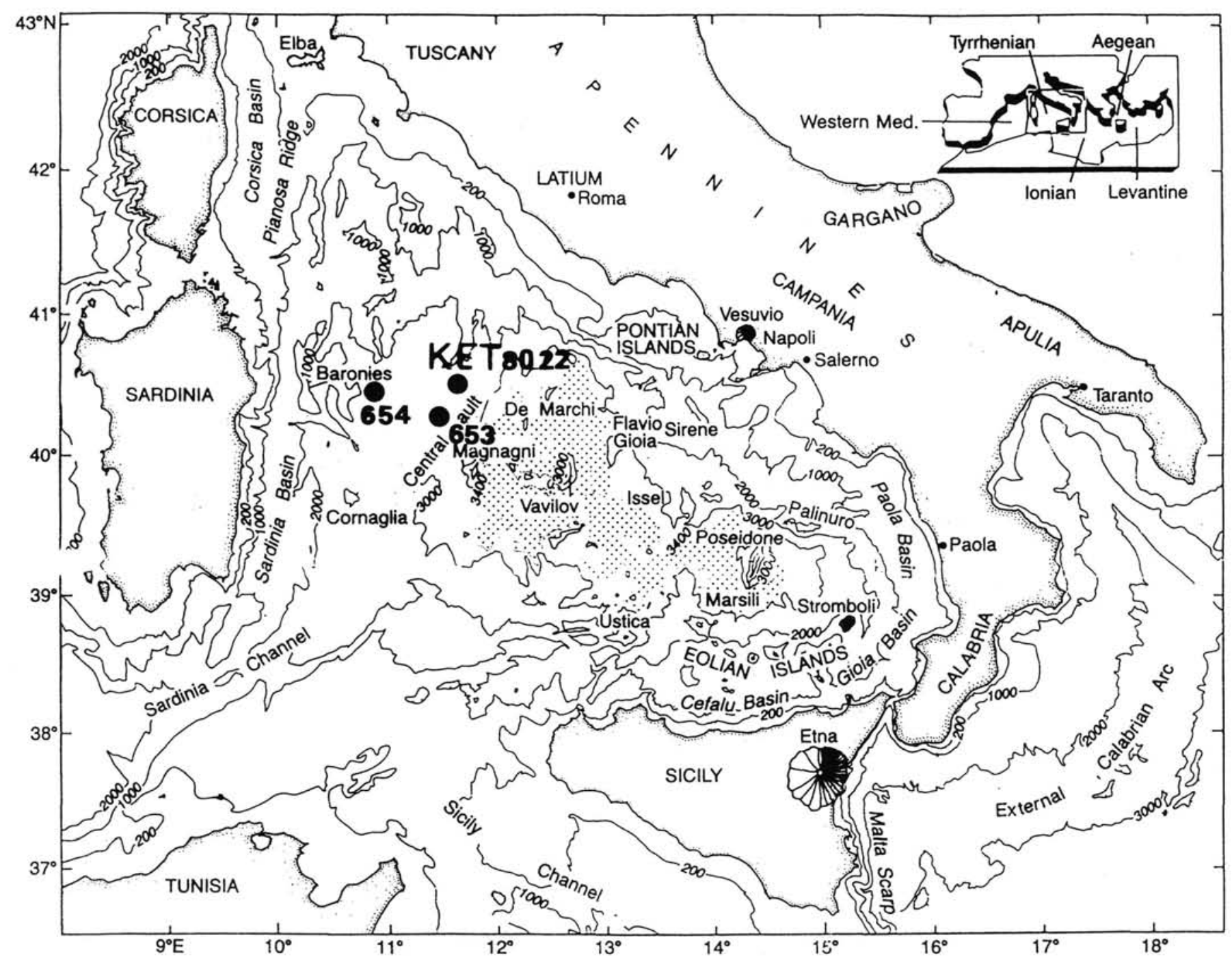

Figure 1. Location of Sites 653 and 654 and Core KET 8022 on a simplified bathymetric map of the Tyrrhenian basin (200-, 1000-, 2000-, and 3000-m bathymetric lines).

Table 2. Compared $\delta^{18} \mathrm{O}$ and $\delta^{13} \mathrm{C}$ values of $G$. bulloides and $G$. falconensis from the same samples at ODP Site 654.

\begin{tabular}{|c|c|c|c|c|c|c|c|}
\hline \multirow{2}{*}{$\begin{array}{l}\text { Core, section, } \\
\text { interval }(\mathrm{cm})\end{array}$} & \multirow{2}{*}{$\begin{array}{l}\text { Depth } \\
\text { (mbsf) }\end{array}$} & \multicolumn{2}{|c|}{ G. bulloides } & \multicolumn{2}{|c|}{ G. falconensis } & \multirow[b]{2}{*}{$\Delta^{18} \mathrm{O}$} & \multirow[b]{2}{*}{$\Delta^{13} \mathrm{C}$} \\
\hline & & $\delta^{18} \mathrm{O}$ & $\delta^{13} \mathrm{C}$ & $\delta^{18} \mathrm{O}$ & $\overline{\delta^{13} \mathrm{C}}$ & & \\
\hline \multicolumn{8}{|l|}{$107-654 \mathrm{~A}-$} \\
\hline $3-4,90$ & 17.40 & - & -0.43 & - & -0.49 & - & -0.04 \\
\hline $16-3,97$ & 140.27 & 1.08 & -0.22 & 1.22 & -0.20 & 0.14 & 0.02 \\
\hline $16-4,37$ & 141.77 & 1.11 & -0.33 & 1.68 & -0.34 & 0.57 & -0.01 \\
\hline $18-5,32$ & 160.96 & - & 0.42 & - & -0.26 & - & -0.68 \\
\hline $18-5,92$ & 161.56 & 0.98 & 0.17 & 1.25 & -0.23 & 0.27 & -0.40 \\
\hline $18-6,32$ & 162.46 & 0.92 & 0.72 & 1.54 & 0.13 & 0.62 & -0.59 \\
\hline $18-6,92$ & 163.02 & - & 0.51 & - & 0.05 & - & -0.46 \\
\hline $21-3,92$ & 188.56 & 0.82 & -0.61 & 0.92 & -1.08 & 0.1 & -0.47 \\
\hline $21-4,35$ & 190.06 & - & 0.10 & - & -0.34 & - & -0.44 \\
\hline $21-5,82$ & 191.56 & 1.61 & 0.36 & 1.69 & 0.12 & 0.08 & -0.24 \\
\hline \multicolumn{3}{|c|}{ Average differences } & & & & 0.30 & -0.33 \\
\hline
\end{tabular}

that modern sediments as well as sediments belonging to substages 4.0 and 5.3 have not been recovered. Substages 3.01-3.13 are not easy to differentiate but are located between the levels 1$2,135 \mathrm{~cm}$, and $2-1,5 \mathrm{~cm}$; the same holds true for substages
$3.31-5.1$, located between levels $2-1,30 \mathrm{~cm}$, and $2-2,30 \mathrm{~cm}$. Substage 5.5 is represented by the adjusted $\delta^{18} \mathrm{O}$ value of $G$. ruber at $2-2,90 \mathrm{~cm}$. A correction factor of +1.42 (calculated on 20 paired analyses of $G$. bulloides and $G$. ruber) was used. The estimated $\delta^{18} \mathrm{O}$ value for substage 5.5 is around +0.85 , a value identical to that reported by Paterne and others for the same substage in Core KET $8022(+0.84)$. The increase in the abundance of $E$. huxleyi around 4 mbsf (Rio et al., this volume) also suggests that the sediments corresponding to substage 5.1 should have been deposited between levels $2-1,60 \mathrm{~cm}$, and the base of Core 1. Some hiatuses therefore do exist in the upper part of the sedimentary sequence of Hole $653 \mathrm{~A}$. This is corroborated by the fact that at Hole $653 \mathrm{~A}$ stage 5.5 is recorded at $6.1 \mathrm{mbsf}$ while at the nearby location of Core KET 8022 it is recorded deeper at 7.5 mbsf. Isotope stage 10 is well pronounced and corresponds, together with stage 2.2 , to the highest $\delta^{18} \mathrm{O}$ values of the entire record $(+3.37 \%)$. This suggests that the glacial stages 8 and 6 have not been sampled at the level of their maximal $\delta^{18} \mathrm{O}$ values (Fig. 3B).

Disturbance problems result in anomalous thickness of the sediments at the level of Core 4 (with important slump folds in Sections 2, 3, and 5) and are responsible for the atypical morphology of isotopic stage 12 (with additional isotopic peaks be- 
C. VERGNAUD GRAZZINI, J. F. SALIÈGE, M. J. URRUTIAGUER, A. IANNACE

Table 3. Oxygen and carbon isotope data for Hole 653A. Depths have been calculated using the data from the corresponding site chapter (Kastens et al., 1987).

\begin{tabular}{|c|c|c|c|c|c|c|}
\hline $\begin{array}{l}\text { Levels } \\
\text { in the cores }\end{array}$ & $\begin{array}{l}\text { Depths } \\
\text { (mbsf) }\end{array}$ & $\begin{array}{c}\text { Age } \\
1000 \mathrm{yr}\end{array}$ & $\begin{array}{c}\delta^{18} \mathrm{O} \% 0 \\
\text { G. bulloides }\end{array}$ & $\begin{array}{l}\delta^{13} \mathrm{C} \% \\
\text { G. bulloides }\end{array}$ & $\begin{array}{l}\delta^{18} \mathrm{O} \% 0 \\
\text { G. ruber }\end{array}$ & $\begin{array}{l}\delta^{13} \mathrm{C} \% 0 \\
\text { G. ruber }\end{array}$ \\
\hline $1-1$, top & 0 & 8.5 & & & 0.89 & 0.96 \\
\hline $1-1,5$ & 0.05 & 8.66 & 0.55 & -0.85 & & \\
\hline $1-1,30$ & 0.3 & 9.5 & & & 0.61 & 1.72 \\
\hline $1-1,60$ & 0.60 & 10.4 & 2.24 & -0.57 & & \\
\hline $1-1,90$ & 0.9 & 11.4 & 3.05 & -0.48 & & \\
\hline $1-1,101$ & 1.01 & 11.6 & 3.31 & -1.16 & & \\
\hline $1-1,120$ & 1.20 & 14.7 & 2.82 & -1.67 & & \\
\hline $1-1,135$ & 1.35 & 15.6 & 3.45 & -0.53 & & \\
\hline $1-2,5$ & 1.55 & 18.1 & 4.14 & -0.72 & & \\
\hline $1-2,25$ & 1.75 & 19.3 & 3.38 & -0.97 & & \\
\hline $1-2,30$ & 1.80 & 19.6 & 3.31 & -0.92 & & \\
\hline $1-2,90$ & 2.40 & 23.2 & 3.05 & -0.21 & & \\
\hline $1-2,100$ & 2.50 & 26 & 2.03 & -1.74 & & \\
\hline $1-2,120$ & 2.70 & 32 & 2.69 & -1.19 & & \\
\hline $1-2,135$ & 2.85 & 35.7 & 3.03 & -0.68 & & \\
\hline $1-3,25$ & 3.25 & 49 & 3.03 & -0.06 & & \\
\hline $2-1,5$ & 3.75 & 61 & 2.95 & -0.45 & & \\
\hline $2-1,30$ & 4.05 & 69 & 2.69 & -0.44 & & \\
\hline $2-1,90$ & 4.6 & 84 & 2.4 & -0.48 & & \\
\hline $2-1,135$ & 5.05 & 97 & 2.56 & -0.43 & & \\
\hline $2-2,30$ & 5.5 & 110 & 2.31 & -0.24 & & \\
\hline $2-2,90$ & 6.1 & 125 & 0.85 & & -0.57 & 0.91 \\
\hline $2-2,131$ & 6.61 & 131 & 2.33 & -0.49 & & \\
\hline $2-3,4$ & 6.74 & 132 & 3.47 & -0.86 & & \\
\hline $2-3,30$ & 7.0 & 135 & 3.53 & -0.45 & & \\
\hline $2-3,90$ & 7.6 & 150 & 2.9 & -0.78 & & \\
\hline $2-4,4$ & 8.24 & 170 & 2.16 & -0.99 & & \\
\hline $2-4,30$ & 8.5 & 177 & 3.34 & -0.81 & & \\
\hline $2-4,90$ & 9.1 & 196 & 1.33 & -0.85 & & \\
\hline $2-4,131$ & 9.51 & 202 & 2.02 & -0.78 & & \\
\hline $2-5,4$ & 9.74 & 204 & 2.13 & -0.97 & & \\
\hline $2-5,30$ & 10.0 & 206 & 2.42 & -0.74 & & \\
\hline $2-5,90$ & 10.6 & 211 & & & 0.32 & 0.64 \\
\hline $2-6,4$ & 11.24 & 219 & 1.57 & -0.14 & 0.2 & 0.67 \\
\hline $2-6,30$ & 11.5 & 225 & & & -0.28 & 1.21 \\
\hline $2-6,90$ & 12.1 & 237 & 1.52 & -1 & & \\
\hline $2-6,132$ & 12.52 & 245 & 2.2 & -1.16 & & \\
\hline $2-7,30$ & 13.1 & 248 & 2.75 & -0.62 & & \\
\hline $3-1,4$ & 13.24 & 250 & 1.99 & -0.98 & & \\
\hline $3-1,30$ & 13.5 & 252 & 3.24 & -0.56 & & \\
\hline $3-1,88$ & 14.08 & 257 & 2.67 & -1.03 & & \\
\hline $3-1,135$ & 14.55 & 261 & 2.94 & -0.85 & & \\
\hline $3-2,5$ & 14.75 & 263 & 2.76 & -1.17 & & \\
\hline $3-2,30$ & 15.0 & 266 & 3.39 & -1.03 & & \\
\hline $3-2,88$ & 15.58 & 280 & 3.1 & 0.09 & & \\
\hline $3-2,135$ & 16.05 & 291 & 2.88 & -0.44 & & \\
\hline $3-3,5$ & 16.25 & 297 & 2.09 & -1.06 & & \\
\hline $3-3,30$ & 16.5 & 303 & 2.61 & -0.44 & & \\
\hline $3-3,88$ & 17.08 & 309 & 2.25 & -0.6 & & \\
\hline $3-3,135$ & 17.55 & 315 & 2.11 & -0.49 & & \\
\hline $3-4,5$ & 17.75 & 317 & 1.7 & -0.55 & & \\
\hline $3-4,30$ & 18.0 & 319 & 1.72 & -0.12 & & \\
\hline $3-5,5$ & 19.27 & 334 & 3.15 & -0.87 & & \\
\hline $3-5,88$ & 20.08 & 342 & 3.57 & -0.5 & & \\
\hline $3-5,135$ & 20.55 & 347 & 3.6 & -0.67 & & \\
\hline $3-6,5$ & 20.75 & 349 & 2.76 & -0.62 & & \\
\hline $3-6,30$ & 21 & 352 & 3.87 & -0.29 & & \\
\hline $3-6,88$ & 21.58 & 357 & 3.42 & -0.71 & & \\
\hline $3-6,135$ & 22.05 & 362 & 2.8 & -0.35 & & \\
\hline $3-7,5$ & 22.25 & 365 & 1.86 & -0.69 & & \\
\hline $3-7, \mathrm{CC}$ & 22.7 & 370 & 1.83 & -0.65 & & \\
\hline $4-1,9$ & 22.75 & 371 & 0.89 & -0.57 & & \\
\hline $4-1,30$ & 23.0 & 374 & 2.88 & 0.12 & & \\
\hline $4-1,135$ & 24.05 & 387 & 0.92 & -0.64 & & \\
\hline $4-2,5$ & 24.25 & 389 & 2.27 & -0.13 & & \\
\hline $4-2,31$ & 24.251 & 389.5 & 2.38 & -0.05 & & \\
\hline $4-2,90$ & 25.21 & 401 & 1.59 & -0.52 & & \\
\hline $4-2,135$ & 25.55 & 405 & 0.84 & -0.63 & & \\
\hline $4-3,5$ & 25.75 & 407 & 0.94 & -1.25 & -0.96 & 1 \\
\hline $4-3,28$ & 25.98 & 410 & & & -0.59 & 1.23 \\
\hline $4-3,135$ & 27.05 & 423 & & & 0.14 & 1.05 \\
\hline $4-4,5$ & 27.25 & 427 & 3.19 & -0.47 & & \\
\hline $4-4,30$ & 27.5 & 431 & 1.95 & -0.63 & & \\
\hline $4-4,90$ & 28.1 & 442 & 1.87 & -0.79 & & \\
\hline $4-4,135$ & 28.55 & 450 & 3.04 & -1.07 & & \\
\hline $4-5,5$ & 28.75 & 453 & 2.79 & -0.92 & 1.07 & 0.63 \\
\hline
\end{tabular}


Table 3 (continued).

\begin{tabular}{|c|c|c|c|c|c|c|}
\hline $\begin{array}{l}\text { Levels } \\
\text { in the cores }\end{array}$ & $\begin{array}{l}\text { Depths } \\
\text { (mbsf) }\end{array}$ & $\begin{array}{l}\text { Age } \\
1000 \mathrm{yr}\end{array}$ & $\begin{array}{c}\delta^{18} \mathrm{O} \% 0 \\
\text { G. bulloides }\end{array}$ & $\begin{array}{c}\delta^{13} \mathrm{C} \%_{0} \\
\text { G. bulloides }\end{array}$ & $\begin{array}{l}\delta^{18} \mathrm{O} \% 0 \\
\text { G. ruber }\end{array}$ & $\begin{array}{l}\delta^{13} \mathrm{C} \% 0 \\
\text { G. ruber }\end{array}$ \\
\hline $4-5,30$ & 29.0 & 457 & 3.3 & -0.5 & & \\
\hline $4-5,135$ & 30.05 & 463 & 2.05 & -0.67 & & \\
\hline $4-6,5$ & 30.25 & 464 & 2.84 & -0.5 & & \\
\hline $4-7,5$ & 31.75 & 470 & 1.86 & -0.72 & & \\
\hline $4-7,30$ & 32.0 & 471 & 2.81 & -0.67 & & \\
\hline $5-1,5$ & 32.25 & 472 & 3.28 & -0.53 & & \\
\hline $5-1,30$ & 32.5 & 473 & 3.23 & -0.37 & & \\
\hline $5-1,90$ & 33.1 & 475 & 3.11 & -0.37 & & \\
\hline $5-1,135$ & 33.55 & 477 & 2.88 & -0.26 & & \\
\hline $5-2,30$ & 34.0 & 486 & 1.88 & -0.45 & & \\
\hline $5-2,90$ & 34.6 & 503 & 2.36 & 0.06 & & \\
\hline $5-3,5$ & 35.25 & 522 & 1.63 & -1.45 & & \\
\hline $5-3,30$ & 35.5 & 526 & 2.92 & -0.32 & & \\
\hline $5-3,92$ & 36.12 & 534 & 1.72 & -0.59 & & \\
\hline $5-3,135$ & 36.55 & 540 & 2.57 & -0.18 & & \\
\hline $5-4,5$ & 36.75 & 542 & 2.06 & -0.89 & & \\
\hline $5-4,92$ & 37.62 & 553 & 2.76 & -0.93 & & \\
\hline $5-4,135$ & 38.05 & 559 & 2.71 & -0.42 & & \\
\hline $5-5,5$ & 38.25 & 561 & 2.57 & -0.39 & & \\
\hline $5-5,30$ & 38.5 & 565 & 1.66 & -0.74 & & \\
\hline $5-5,92$ & 39.12 & 578 & 2.74 & -0.57 & & \\
\hline $5-6,5$ & 39.75 & 592 & 1.59 & -0.89 & 0.99 & 1.02 \\
\hline $5-6,30$ & 40.0 & 598 & 1.81 & -0.53 & & \\
\hline $5-6,92$ & 40.62 & 609 & 1.89 & -0.7 & & \\
\hline $5-6,132$ & 41.05 & 621 & 2.7 & -0.99 & & \\
\hline $5-7,5$ & 41.25 & 626 & 2.22 & -0.79 & & \\
\hline $6-1,5$ & 41.65 & 635 & 2.88 & -1.09 & & \\
\hline $6-1,30$ & 41.9 & 641 & 3.29 & -0.96 & & \\
\hline $6-1,90$ & 42.5 & 656 & 2.92 & -0.65 & & \\
\hline $6-2,5$ & 43.15 & 669 & 1.27 & -1.01 & & 0.39 \\
\hline $6-2,135$ & 44.435 & 693 & 2.9 & -0.89 & & \\
\hline $6-3,5$ & 44.65 & 698 & 2.77 & -0.78 & & \\
\hline $6-3,30$ & 44.9 & 703 & 2.42 & -0.46 & 0.58 & 0.39 \\
\hline $6-3,90$ & 45.5 & 715 & 2.81 & -0.73 & & \\
\hline $6-4,5$ & 46.15 & 726 & 2.23 & -0.25 & & \\
\hline $6-4,90$ & 47.0 & 729 & 2.67 & -0.37 & & \\
\hline $6-5,5$ & 47.65 & 732 & 2.05 & -0.39 & & \\
\hline $6-5,90$ & 48.5 & 735 & 2.48 & -0.95 & & \\
\hline $6-6,3$ & 49.15 & 747 & 3.12 & -0.62 & 1.87 & 0.83 \\
\hline $6-6,30$ & 49.4 & 752 & 2.43 & -0.46 & & \\
\hline $7-1,5$ & 51.05 & 786 & 3.16 & -0.77 & 1.63 & 0.74 \\
\hline $7-1,30$ & 51.3 & 791 & 2.82 & -0.86 & & \\
\hline $7-1,90$ & 51.9 & 804 & 2.53 & -0.26 & & \\
\hline $7-1,135$ & 52.35 & 813 & 2.62 & -0.81 & & \\
\hline $7-2,5$ & 52.55 & 817 & 2.29 & -0.6 & 0.65 & 0.93 \\
\hline $7-2,30$ & 52.8 & 818 & 3.57 & -0.55 & & \\
\hline $7-2,135$ & 53.85 & 844 & 3.45 & -0.93 & & \\
\hline $7-3,95$ & 54.95 & 867 & 3.08 & -1.01 & & \\
\hline $7-4,5$ & 55.55 & 880 & 1.49 & -1.08 & & \\
\hline $7-4,90$ & 56.4 & 897 & 2.43 & -0.41 & & \\
\hline $7-5-5$ & 57.05 & 911 & 1.4 & -0.81 & & \\
\hline $7-5,30$ & 57.3 & 916 & 2.31 & -0.62 & & \\
\hline $7-5,135$ & 58.35 & 938 & 2.07 & -0.06 & & \\
\hline $7-6,5$ & 58.55 & 942 & 3.08 & -0.55 & & \\
\hline $7-6,30$ & 58.8 & 947 & 1.8 & -0.83 & & \\
\hline $7-6,90$ & 59.4 & 961 & 2.01 & -0.3 & & \\
\hline $7-6,132$ & 59.82 & 970 & 2.07 & -0.31 & & \\
\hline $7-7,5$ & 60.05 & 976 & 1.96 & -0.96 & 0.34 & 0.99 \\
\hline $7-7,30$ & 60.3 & 981 & 2.28 & -0.62 & & \\
\hline $8-1,6$ & 60.56 & 987 & 1.97 & -0.99 & -0.33 & 0.45 \\
\hline $8-1,90$ & 61.4 & 1007 & 2.64 & -1.21 & & \\
\hline $8-1,135$ & 61.85 & 1017 & 2.44 & -0.95 & & \\
\hline $8-2,6$ & 62.05 & 1022 & 2.57 & -0.78 & 1.29 & 1.12 \\
\hline $8-2,30$ & 62.3 & 1028 & 2.32 & -0.53 & & \\
\hline $8-2,135$ & 63.35 & 1052 & 1.85 & -0.73 & & \\
\hline $8-3,5$ & 63.55 & 1056 & 2.83 & -0.85 & 0.66 & 1.06 \\
\hline $8-3,34$ & 63.84 & 1063 & 2.22 & -0.86 & & \\
\hline $8-3,135$ & 64.85 & 1086 & 1.52 & -0.93 & & \\
\hline $8-4,7$ & 65.07 & 1091 & 1.91 & -0.86 & -0.57 & 1.23 \\
\hline $8-4,30$ & 65.3 & 1097 & 2.9 & -0.64 & & \\
\hline $8-4,90$ & 65.9 & 1112 & 2.54 & -0.14 & & \\
\hline $8-5,9$ & 66.59 & 1130 & 2.12 & -0.91 & 0.39 & 1.08 \\
\hline $8-5,30$ & 66.8 & 1136 & 2.71 & -0.51 & & \\
\hline $8-5,90$ & 67.4 & 1151 & 2.45 & -0.01 & & \\
\hline $8-6,5$ & 68.05 & 1169 & 2.05 & -0.56 & -0.01 & 1.18 \\
\hline $8-6,30$ & 68.3 & 1175 & 2.57 & -0.57 & & \\
\hline $8-7,6$ & 69.56 & 1208 & 1.4 & -0.82 & 0.36 & 0.81 \\
\hline
\end{tabular}


Table 3 (continued).

\begin{tabular}{|c|c|c|c|c|c|c|}
\hline $\begin{array}{l}\text { Levels } \\
\text { in the cores }\end{array}$ & $\begin{array}{l}\text { Depths } \\
\text { (mbsf) }\end{array}$ & $\begin{array}{c}\text { Age } \\
1000 \mathrm{yr}\end{array}$ & $\begin{array}{c}\delta^{18} \mathrm{O} \% 0 \\
\text { G. bulloides }\end{array}$ & $\begin{array}{c}\delta^{13} \mathrm{C} \%_{0} \\
\text { G. bulloides }\end{array}$ & $\begin{array}{l}\delta^{18} \mathrm{O} \% \\
\text { G. ruber }\end{array}$ & $\begin{array}{l}\delta^{13} \mathrm{C} \% \\
\text { G. ruber }\end{array}$ \\
\hline $8-7,30$ & 69.8 & 1214 & 2.83 & -0.25 & & \\
\hline $9-1,5$ & 70.05 & 1221 & 2.14 & -0.42 & 0.9 & 0.7 \\
\hline $9-1,30$ & 70.3 & 1228 & & & -0.42 & 0.92 \\
\hline $9-1,90$ & 70.9 & 1243 & 2.28 & -0.25 & & \\
\hline $9-1,135$ & 71.135 & 1250 & & & -0.47 & 0.97 \\
\hline $9-2,5$ & 71.55 & 1260 & 1.8 & -0.74 & 0.43 & 1.15 \\
\hline $9-2,30$ & 71.8 & 1267 & 2.25 & -0.67 & & \\
\hline $9-2,90$ & 72.4 & 1283 & & & -0.99 & 0.85 \\
\hline $9-2,135$ & 72.85 & 1295 & & & -0.39 & 1.33 \\
\hline $9-3,5$ & 73.05 & 1300 & 1.66 & -0.45 & 0.73 & 0.82 \\
\hline $9-3,32$ & 73.32 & 1307 & 2.21 & -0.5 & & \\
\hline $9-3,92$ & 73.92 & 1323 & 2.27 & -0.75 & & \\
\hline $9-3,133$ & 74.33 & 1333 & 1.85 & -0.13 & 0.65 & 0.42 \\
\hline $9-4,5$ & 74.55 & 1339 & 2.16 & -0.07 & 0.5 & 0.75 \\
\hline $9-4,30$ & 74.8 & 1346 & 2.81 & -0.22 & & \\
\hline $9-4,90$ & 75.4 & 1362 & & & 0.87 & 1.44 \\
\hline $9-4,135$ & 75.85 & 1373 & 2.47 & 0.04 & & \\
\hline $9-5,5$ & 76.05 & 1379 & 2.56 & -0.18 & 1.9 & 1.29 \\
\hline $9-5,30$ & 76.3 & 1385 & 2.34 & 0.04 & & \\
\hline $9-5,90$ & 76.9 & 1401 & 2.2 & 0.31 & & \\
\hline $9-5,135$ & 77.35 & 1413 & 2.34 & 0.04 & & \\
\hline $9-6,5$ & 77.55 & 1418 & 1.84 & -0.32 & 1.15 & 1.44 \\
\hline $9-6,30$ & 77.8 & 1425 & 2.07 & 0.27 & & \\
\hline $9-7,5$ & 79.05 & 1457 & 1.47 & -0.47 & 0.04 & 1.35 \\
\hline $9-7,30$ & 79.3 & 1464 & 1.7 & 0.42 & & \\
\hline $10-1,5$ & 79.65 & 1473 & 1.68 & -0.26 & 1.14 & 1.29 \\
\hline $10-1,30$ & 79.9 & 1480 & 2.36 & -0.11 & & \\
\hline $10-1,90$ & 80.5 & 1495 & 1.75 & -0.17 & & \\
\hline $10-1,135$ & 80.95 & 1506 & 1.74 & -0.11 & 0.29 & 0.99 \\
\hline $10-2,5$ & 81.15 & 1511 & 1.25 & -1.16 & 0.36 & 0.66 \\
\hline $10-2,30$ & 81.4 & 1518 & 2.86 & -0.44 & & \\
\hline $10-2,90$ & 82.0 & 1533 & 1.22 & -1.03 & & \\
\hline $10-2,135$ & 82.45 & 1544 & 2.07 & 0.21 & & \\
\hline $10-3,5$ & 82.65 & 1549 & 1.44 & -0.72 & 0.04 & 1.68 \\
\hline $10-3,30$ & 82.9 & 1555 & 1.88 & -0.11 & & \\
\hline $10-3,90$ & 83.5 & 1570 & 1.64 & 0.59 & G. elongatus & G. elongatus \\
\hline $10-3,135$ & 83.95 & 1581 & & & 0.29 & 1.2 \\
\hline $10-4,5$ & 84.15 & 1586 & 1.9 & -0.39 & 0.33 & 0.93 \\
\hline $10-4,30$ & 84.4 & 1592 & 2.7 & 0.31 & & \\
\hline $10-4,90$ & 85.0 & 1607 & 1.95 & 0.15 & & \\
\hline $10-4,135$ & 85.45 & 1618 & & & -0.52 & 1.8 \\
\hline $10-5,5$ & 85.65 & 1622 & 1.72 & -0.46 & -0.31 & 1.43 \\
\hline $10-5,30$ & 85.9 & 1627 & 2.1 & 0.89 & & \\
\hline $10-5,90$ & 86.5 & 1685 & 1.72 & 0.68 & & \\
\hline $10-5,135$ & 86.95 & 1715 & 2.25 & 0.31 & & \\
\hline $10-6,5$ & 87.15 & 1728 & 2.46 & 0.38 & 0.67 & 1.27 \\
\hline $10-6,30$ & 87.4 & 1744 & 2.01 & 0.43 & & \\
\hline $10-6,90$ & 88.0 & 1784 & & & 1 & 1.97 \\
\hline $10-6,135$ & 88.45 & 1813 & & & & \\
\hline $11-1,5$ & 89.05 & 1839 & 2.05 & 0.55 & 0.19 & 1.49 \\
\hline $11-1,30$ & 89.3 & 1846 & & & 0.83 & 1.75 \\
\hline $11-1,90$ & 89.9 & 1863 & & & -0.56 & 1.35 \\
\hline $11-2,5$ & 90.55 & 1880 & 1.44 & 0.48 & -0.22 & 1.33 \\
\hline $11-2,30$ & 90.8 & 1887 & & & 0.05 & 1.65 \\
\hline $11-2,90$ & 91.4 & 1904 & & & -0.35 & 1.44 \\
\hline $11-2,135$ & 91.85 & 1916 & & & -0.44 & 0.84 \\
\hline $11-3,5$ & 92.05 & 1922 & 2.15 & 0.02 & 0.83 & 1.3 \\
\hline $11-330$ & 92.3 & 1929 & 2.41 & 0.48 & & \\
\hline $11-4,5$ & 93.55 & 1963 & 1.35 & 0.39 & 0.32 & 1.33 \\
\hline $11-4,30$ & 93.8 & 1970 & & & -0.08 & 2.07 \\
\hline $11-4,90$ & 94.4 & 1987 & & & 0.72 & 1.51 \\
\hline $11-4,135$ & 94.85 & 1999 & & & 0.23 & 1.74 \\
\hline $11-5-5$ & 95.05 & 2004 & 1.83 & 0.06 & -0.82 & 0.8 \\
\hline $11-5.30$ & 95.3 & 2011 & 1.74 & 0.53 & 0.06 & 1.75 \\
\hline $11-5,90$ & 95.9 & 2028 & & & 0.05 & 1.91 \\
\hline $11-5,135$ & 96.35 & 2040 & & & 0.56 & 1.99 \\
\hline $11-6,5$ & 96.55 & 2046 & 1.71 & 0.45 & 0.92 & 1.54 \\
\hline $11-6,30$ & 96.8 & 2053 & & & 0.46 & 1.62 \\
\hline $11-6,135$ & 97.85 & 2082 & 1.96 & 0.36 & & \\
\hline $11-7,5$ & 98.05 & 2087 & 1.84 & 0.11 & 0.2 & 1.49 \\
\hline $11-7,30$ & 98.2 & 2091 & 1.52 & -0.35 & & \\
\hline $12-1,7$ & 98.27 & 2091 & 2.18 & 0.35 & & \\
\hline $12-1,30$ & 98.5 & 2100 & & & 0.07 & 1.19 \\
\hline $12-1,90$ & 99.1 & 2113 & & & -0.26 & 0.87 \\
\hline $12-1,135$ & 99.55 & 2124 & 2.06 & 0.31 & & \\
\hline
\end{tabular}


Table 3 (continued).

\begin{tabular}{|c|c|c|c|c|c|c|}
\hline $\begin{array}{l}\text { Levels } \\
\text { in the cores }\end{array}$ & $\begin{array}{l}\text { Depths } \\
\text { (mbsf) }\end{array}$ & $\begin{array}{l}\text { Age } \\
1000 \mathrm{yr}\end{array}$ & $\begin{array}{c}\delta^{18} \mathrm{O} \%_{0} \\
\text { G. bulloides }\end{array}$ & $\begin{array}{c}\delta^{13} \mathrm{C} \%_{0} \\
\text { G. bulloides }\end{array}$ & $\begin{array}{l}\delta^{18} \mathrm{O} \%_{0} \\
\text { G. ruber }\end{array}$ & $\begin{array}{l}\delta^{13} \mathrm{C} \%_{0} \\
\text { G. ruber }\end{array}$ \\
\hline $12-2,5$ & 99.75 & 2128 & 2.62 & 0.56 & 0.76 & 1.73 \\
\hline $12-2,30$ & 100.0 & 2134 & & & 0.26 & 1.77 \\
\hline $12-2,90$ & 100.6 & 2148 & & & -0.03 & 0.44 \\
\hline $12-2,135$ & 101.05 & 2158 & -0.6 & 0.88 & & \\
\hline $12-3,5$ & 101.25 & 2163 & 1.63 & 0.09 & -0.31 & 1.18 \\
\hline $12-3,30$ & 101.5 & 2171 & & & -0.24 & 1.31 \\
\hline $12-4,5$ & 102.75 & 2198 & 1.7 & 0.58 & -0.07 & 1.4 \\
\hline $12-4,135$ & 104.05 & 2228 & & & -0.54 & 1.41 \\
\hline $12-5,5$ & 104.25 & 2232 & 1.8 & 0.48 & -0.37 & 1.53 \\
\hline $12-5,30$ & 104.5 & 2238 & & & 0.15 & 1.54 \\
\hline $12-5,90$ & 105.1 & 2252 & & & 0.52 & 1.66 \\
\hline $12-5,135$ & 105.55 & 2262 & & & 0.46 & 1.67 \\
\hline $12-6,5$ & 105.75 & 2267 & 2.43 & 0.73 & 0.41 & 1.79 \\
\hline $12-6,30$ & 106.0 & 2273 & & & 0.12 & 2.01 \\
\hline $12-6,90$ & 106.6 & 2287 & & & -0.3 & 1.91 \\
\hline $12-7,5$ & 107.25 & 2302 & 1.2 & 0.36 & 0.01 & 1.45 \\
\hline $13-1,135$ & 108.55 & 2332 & & & 0.17 & 1.78 \\
\hline $13-2,5$ & 109.25 & 2348 & 0.97 & -0.24 & -0.56 & 1.42 \\
\hline $13-2,30$ & 109.5 & 2354 & & & -1.14 & 0.8 \\
\hline $13-2,90$ & 110.1 & 2367 & 1.75 & 0.52 & & \\
\hline $13-2,135$ & 110.55 & 2378 & & & -0.5 & 1.07 \\
\hline $13-3,5$ & 110.75 & 2382 & 2.01 & 0.09 & 0.13 & 1 \\
\hline $13-3,30$ & 111.0 & 2388 & 0.9 & -0.3 & & \\
\hline $13-3,90$ & 111.6 & 2402 & 0.78 & -0.13 & & \\
\hline $13-3,135$ & 112.05 & 2414 & & & -0.28 & 1.45 \\
\hline $13-4,5$ & 112.25 & 2419 & 1.4 & 0.25 & & \\
\hline $13-4,30$ & 112.5 & 2426 & & & -0.09 & 1.38 \\
\hline $13-4,90$ & 113.1 & 2441 & 0.99 & -0.5 & & \\
\hline $13-4,135$ & 113.55 & 2453 & & & 0 & 1.24 \\
\hline $13-5,5$ & 113.75 & 2458 & 2.09 & -0.04 & 0.66 & 1.11 \\
\hline $13-5,30$ & 114.0 & 2464 & 1.46 & -0.1 & & \\
\hline $13-5,90$ & 114.6 & 2480 & 1.58 & -0.32 & & \\
\hline $13-5,135$ & 115.05 & 2492 & & & -0.02 & 1.37 \\
\hline $13-6,5$ & 115.25 & 2497 & 1.52 & 0.16 & 0.89 & 1.38 \\
\hline $13-6,30$ & 115.5 & 2503 & & & 0.02 & 1.72 \\
\hline $13-6,90$ & 116.1 & 2519 & & & 0.67 & 1.19 \\
\hline $13-6,135$ & 116.55 & 2530 & & & 0.41 & 1.62 \\
\hline $14-1,5$ & 117.35 & 2551 & 1.56 & 0.07 & & \\
\hline $14-1,30$ & 117.6 & 2558 & & & -0.14 & 1.89 \\
\hline $14-1,90$ & 118.2 & 2573 & & & 0.27 & 1.35 \\
\hline $14-1,135$ & 118.65 & 2585 & & & -0.29 & 1.5 \\
\hline $14-2,5$ & 118.85 & 2590 & 1.01 & 0.01 & & \\
\hline $14-2,30$ & 119.15 & 2598 & & & 0.33 & 1.58 \\
\hline $14-2,90$ & 119.75 & 2615 & & & 0.08 & 1.21 \\
\hline $14-2,135$ & 120.2 & 2627 & & & -0.4 & 1.78 \\
\hline $14-3,5$ & 120.35 & 2632 & 0.49 . & 0.15 & & \\
\hline $14-3,30$ & 120.6 & 2639 & & & -0.51 & 1.34 \\
\hline $14-3,90$ & 121.2 & 2658 & & & -0.64 & 1.94 \\
\hline $14-3,135$ & 121.65 & 2668 & 0.75 & 0.23 & & \\
\hline $14-4,5$ & 121.85 & 2674 & 0.6 & -0.44 & & \\
\hline $14-4,30$ & 122.1 & 2681 & & & -0.83 & 1.24 \\
\hline $14-4,90$ & 122.7 & 2698 & 1.28 & -0.22 & & \\
\hline $14-4,135$ & 123.15 & 2711 & & & 0.08 & 1.73 \\
\hline $14-5,5$ & 123.35 & 2717 & 0.99 & -0.49 & & \\
\hline $14-5,30$ & 123.6 & 2724 & & & 0 & 1.2 \\
\hline $14-5,90$ & 124.2 & 2741 & & & -0.93 & 1.33 \\
\hline $14-5,135$ & 124.65 & 2753 & 1.21 & -0.22 & & \\
\hline $14-6,30$ & 125.1 & 2766 & & & -0.16 & 0.83 \\
\hline $14-6,90$ & 125.7 & 2783 & & & -0.54 & 0.98 \\
\hline $14-6,135$ & 126.15 & 2796 & & & -0.68 & 1.44 \\
\hline $14-7,5$ & 126.35 & 2802 & 1.54 & 0.03 & & \\
\hline $14-7,30$ & 126.6 & 2809 & & & -0.22 & 1.41 \\
\hline $15-1,05$ & 126.72 & 2812 & 1.2 & -0.38 & & \\
\hline $15-1,30$ & 126.97 & 2819 & & & 0 & 1.17 \\
\hline $15-1,90$ & 127.57 & 2836 & & & -0.22 & 1.38 \\
\hline $15-1,135$ & 128.02 & 2849 & & & -1.28 & 0.7 \\
\hline $15-2,5$ & 128.15 & 2853 & 1.54 & -0.33 & & \\
\hline $15-2,90$ & 129.0 & 2877 & & & -1.02 & 1.09 \\
\hline $15-2,135$ & 129.45 & 2889 & & & -0.37 & 1.47 \\
\hline $15-3,5$ & 129.65 & 2895 & 1.18 & -0.61 & & \\
\hline $15-3,30$ & 129.9 & 2902 & 0.84 & -0.13 & & \\
\hline $16-15$ & 136.25 & 3078 & 1.11 & -0.29 & & \\
\hline $16-1,30$ & 136.5 & 3083 & & & -0.02 & 1.45 \\
\hline $16-1,90$ & 137.1 & 3094 & & & -0.24 & 1.25 \\
\hline $16-1,135$ & 137.55 & 3103 & 0.86 & & & \\
\hline
\end{tabular}


C. VERGNAUD GRAZZINI, J. F. SALIĖGE, M. J. URRUTIAGUER, A. IANNACE

Table 3 (continued).

\begin{tabular}{|c|c|c|c|c|c|c|}
\hline $\begin{array}{l}\text { Levels } \\
\text { in the cores }\end{array}$ & $\begin{array}{l}\text { Depths } \\
\text { (mbsf) }\end{array}$ & $\begin{array}{l}\text { Age } \\
1000 \mathrm{yr}\end{array}$ & $\begin{array}{c}\delta^{18} \mathrm{O} \% 0 \\
\text { G. bulloides }\end{array}$ & $\begin{array}{c}\delta^{13} \mathrm{C} \%_{0} \\
\text { G. bulloides }\end{array}$ & $\begin{array}{l}\delta^{18} \mathrm{O} \% 0 \\
\text { G. ruber }\end{array}$ & $\begin{array}{l}\delta^{13} \mathrm{C} \% 0 \\
\text { G. ruber }\end{array}$ \\
\hline $16-2,5$ & 137.65 & 3104 & 1.29 & -0.75 & & \\
\hline $16-2,30$ & 137.9 & 3109 & 1.24 & -0.07 & & \\
\hline $16-2,90$ & 138.5 & 3120 & & & -0.59 & 1.09 \\
\hline $16-2,135$ & 138.95 & 3129 & 1 & -0.01 & & \\
\hline $16-3,5$ & 139.15 & 3133 & 0.85 & -0.69 & & \\
\hline $16-3,30$ & 139.4 & 3137 & & & -0.95 & 0.92 \\
\hline $16-3,90$ & 140.0 & 3149 & & & -0.23 & 1.1 \\
\hline $16-4,5$ & 140.65 & 3161 & 0.12 & -0.56 & & \\
\hline $16-4,90$ & 141.5 & 3177 & & & -0.28 & 1.17 \\
\hline $16-4-135$ & 141.95 & 3188 & & & -0.8 & 0.97 \\
\hline $16-5,5$ & 142.15 & 3189 & 1.08 & -0.54 & & \\
\hline $16-5,30$ & 142.4 & 3194 & 1.11 & -0.11 & & \\
\hline $16-5,90$ & 143.0 & 3205 & 0.86 & -0.08 & & \\
\hline $16-6,5$ & 143.65 & 3218 & 0.53 & -0.56 & & \\
\hline $16-6,30$ & 143.9 & 3222 & & & -1.28 & 0.65 \\
\hline $16-6,90$ & 144.5 & 3224 & & & -0.93 & 0.14 \\
\hline $17-1,5$ & 145.65 & 3255 & 1.36 & -0.02 & & \\
\hline $17-1,30$ & 145.9 & 3260 & -0.25 & 1.38 & & \\
\hline $17-1,90$ & 146.5 & 3271 & 0.62 & 0.48 & & \\
\hline $17-1,135$ & 146.95 & 3278 & & & -0.9 & 1.41 \\
\hline $17-2,5$ & 147.15 & 3282 & 1.55 & 0.47 & & \\
\hline $17-2,30$ & 147.4 & 3286 & 0.53 & 0.36 & & \\
\hline $17-2,90$ & 148.0 & 3295 & 0.74 & 0.31 & -0.46 & 1.41 \\
\hline $17-2,135$ & 148.45 & 3302 & 0.67 & 0.05 & & \\
\hline $17-3,5$ & 148.65 & 3306 & 0.6 & 0.71 & & \\
\hline $17-3,30$ & 148.9 & 3310 & 0.8 & 0.21 & & \\
\hline $17-3,90$ & 149.5 & 3319 & & & -0.87 & 1.65 \\
\hline $17-3,135$ & 149.95 & 3327 & 0.59 & 0.2 & & \\
\hline $17-4,30$ & 150.4 & 3334 & 0.09 & & & \\
\hline $17-4,90$ & 151.0 & 3343 & & & -1.03 & 1.67 \\
\hline $17-4,135$ & 151.45 & 3351 & & & -1.22 & 1.11 \\
\hline $17-5,5$ & 151.65 & 3354 & 0.65 & -0.23 & & \\
\hline $17-5,30$ & 151.9 & 3358 & 0.89 & 0.21 & -0.87 & 1.73 \\
\hline $17-5,90$ & 152.5 & 3380 & & & -0.23 & 1.46 \\
\hline $17-6,5$ & 152.65 & 3386 & 1.22 & 0.55 & G. elongatus & G. obliquus \\
\hline $17-6,30$ & 152.9 & 3396 & & & -0.62 & 1.41 \\
\hline $18-1,05$ & 155.25 & 3490 & 1.39 & 0.1 & & \\
\hline $18-1,32$ & 155.57 & 3502 & & & -0.31 & 1.36 \\
\hline $18-1,90$ & 156.15 & 3522 & & & -1.46 & 1.18 \\
\hline $18-1,135$ & 156.6 & 3529 & & & -0.76 & 1.47 \\
\hline $18-2,30$ & 157.0 & 3535 & & & -0.32 & 0.83 \\
\hline $18-2,135$ & 157.05 & 3544 & & & -0.86 & 1.28 \\
\hline $18-2,90$ & 157.6 & 3551 & & & -1.4 & 0.8 \\
\hline $18-3,30$ & 158.5 & 3562 & & & -1.73 & 0.77 \\
\hline $18-3,90$ & 159.1 & 3577 & & & -0.92 & 1.43 \\
\hline $18-3,135$ & 159.55 & 3588 & & & -0.42 & 1.29 \\
\hline $18-4,30$ & 159.95 & 3598 & & & -0.47 & 1.5 \\
\hline $18-4,90$ & 160.55 & 3613 & & & -1.45 & 1.17 \\
\hline $18-4,135$ & 161.0 & 3625 & & & -0.63 & 1.51 \\
\hline $18-5,30$ & 161.45 & 3636 & & & -0.95 & 1.17 \\
\hline $18-5,90$ & 162.05 & 3651 & & & -0.3 & 1.43 \\
\hline $18-5,135$ & 162.5 & 3662 & & & -0.69 & 1.42 \\
\hline $18-6,30$ & 162.95 & 3673 & & & -0.9 & 1.13 \\
\hline $19-1,30$ & 164.4 & 3710 & & & -0.14 & 1.87 \\
\hline $19-1,90$ & 165.0 & 3725 & & & -0.92 & 1.16 \\
\hline $19-1,135$ & 165.45 & 3736 & & & -1.2 & 1.37 \\
\hline $19-2,30$ & 165.9 & 3744 & & & & \\
\hline $19-2,90$ & 166.5 & 3754 & & & -0.82 & 1.57 \\
\hline $19-2-135$ & 166.95 & 3764 & & & G. obliquus & G. obliquus \\
\hline $19 \cdot 3,12$ & 167.27 & 3771 & 0.09 & -0.81 & & \\
\hline $19-3,30$ & 167.45 & 3775 & & & -0.92 & 1.91 \\
\hline $19-3,90$ & 168.05 & 3788 & & & -0.76 & 1.86 \\
\hline $19-3,135$ & 168.5 & 3798 & & & -0.58 & 1.28 \\
\hline $19-4-90$ & 169.55 & 3822 & & & -1.12 & 1.56 \\
\hline $19-5,30$ & 170.65 & 3843 & & & -1.54 & 1.39 \\
\hline $19-5,90$ & 171.25 & 3854 & & & -1.73 & 1.22 \\
\hline $19-5,137$ & 171.72 & 3863 & & & & 1.51 \\
\hline $19-6,30$ & 172.15 & 3872 & & & & \\
\hline $20-1,30$ & 174.0 & 3905 & & & & \\
\hline $20-1,135$ & 175.05 & 3924 & & & -1.18 & 1.23 \\
\hline $20-2,30$ & 175.5 & 3933 & & & -0.21 & 0.82 \\
\hline $20-2,90$ & 176.1 & 3944 & & & -0.87 & 0.96 \\
\hline $20-2,135$ & 176.55 & 3952 & & & -1 & 1.14 \\
\hline $20-3,90$ & 177.6 & 3971 & & & -0.89 & 1.1 \\
\hline $20-3,135$ & 178.05 & 3980 & & & -0.86 & 1.27 \\
\hline $20-4,86$ & 179.1 & 3999 & 0.36 & -0.62 & & \\
\hline
\end{tabular}


Table 3 (continued).

\begin{tabular}{|c|c|c|c|c|c|c|}
\hline $\begin{array}{l}\text { Levels } \\
\text { in the cores }\end{array}$ & $\begin{array}{l}\text { Depths } \\
\text { (mbsf) }\end{array}$ & $\begin{array}{l}\text { Age } \\
1000 \mathrm{yr}\end{array}$ & $\begin{array}{c}\delta^{18} \mathrm{O} \% 0 \\
\text { G. bulloides }\end{array}$ & $\begin{array}{c}\delta^{13} \mathrm{C} \% 0 \\
G . \text { bulloides }\end{array}$ & $\begin{array}{l}\delta^{18} \mathrm{O} \%_{0} \\
G . \text { ruber }\end{array}$ & $\begin{array}{l}\delta^{13} \mathrm{C} \%_{0} \\
\text { G. ruber }\end{array}$ \\
\hline $20-4,125$ & 179.5 & 4007 & 0.94 & -0.33 & & \\
\hline $20-5,31$ & 180.0 & 4016 & & & -1.02 & 0.9 \\
\hline $20-5,135$ & 181.0 & 4034 & 0.11 & -0.52 & & \\
\hline $21-1,30$ & 183.5 & 4080 & 0.43 & -0.16 & & \\
\hline $21-1,90$ & 184.1 & 4091 & -0.19 & 0.21 & & \\
\hline $21-1,134$ & 184.54 & 4099 & 0.6 & -0.49 & & \\
\hline $21-2,30$ & 185.0 & 4108 & & & -0.73 & 1.74 \\
\hline $21-2,134$ & 186.04 & 4127 & & & -0.47 & 1.41 \\
\hline $21-3,90$ & 187.1 & 4147 & & & -0.87 & 1.35 \\
\hline $21-4,30$ & 188.0 & 4187 & & & -1.26 & 1.41 \\
\hline $22-1,30$ & 193.2 & 4290 & 0.05 & -0.74 & -0.69 & 1.14 \\
\hline $22-1,134$ & 194.24 & 4315 & & & -0.57 & 1.43 \\
\hline $23-1,30$ & 202.7 & 4515 & & & -1.12 & 1.53 \\
\hline $23-1,90$ & 203.3 & 4529 & & & -1.64 & 1.34 \\
\hline $23-1,130$ & 203.7 & 4540 & 0.84 & -0.7 & -0.18 & 1.02 \\
\hline $23-2,30$ & 204.3 & 4553 & 0.43 & -0.48 & -0.86 & 1.49 \\
\hline $23-2,90$ & 204.9 & 4567 & & & -0.71 & 1.75 \\
\hline $23-2,130$ & 205.3 & 4577 & 0.32 & -0.69 & -0.62 & 1.41 \\
\hline $23-3,30$ & 205.7 & 4588 & 0.21 & -0.5 & -0.84 & 1.14 \\
\hline $23-3,90$ & 206.3 & 4600 & & & -0.83 & 1.7 \\
\hline $23-3,130$ & 206.7 & 4610 & & & -0.76 & 1.51 \\
\hline $23-4,30$ & 207.2 & 4622 & & & -1.28 & 1.22 \\
\hline $23-4,90$ & 207.8 & 4636 & -0.07 & -0.26 & -0.94 & 0.7 \\
\hline $23-4,130$ & 208.2 & 4645 & -0.25 & -0.96 & -1.52 & 0.92 \\
\hline $23-5,90$ & 209.3 & 4671 & 1.13 & 0.08 & -1.31 & 1.17 \\
\hline $23-6,30$ & 210.2 & 4691 & & & -0.81 & 0.94 \\
\hline $23-6,90$ & 210.8 & 4704 & 1.28 & -0.64 & -1.51 & 1.49 \\
\hline $23-6,130$ & 211.2 & 4713 & 0.8 & -0.29 & -0.47 & 1.34 \\
\hline $23-7,30$ & 211.7 & 4724 & 0.74 & 0.17 & -0.43 & 0.96 \\
\hline
\end{tabular}

ing an artifact).The identification of this stage is corroborated by the last occurrence datum of Pseudoemiliana lacunosa between the levels $4-5,25 \mathrm{~cm}$, and 4-5, $60 \mathrm{~cm}$, near $29.12 \mathrm{mbsf}$ (Rio et al., this volume).

\section{The Middle Pleistocene (33.5-53 mbsf, 0.45-0.8 Ma)}

In this part of the record isotopic stages 13 through 22 should be recognized. Between 52 and 53 mbsf (Core 7, Section 2 ) isotopic stage 22 appears well marked as in all open ocean records (Figs. 3B, 3C). Other easily identifiable stages are isotopic stages $13,14,15$, and 16 .

Isotope stage 14 , near $38 \mathrm{mbsf}$, corresponds to less positive values than stages 12 or 16 and is bracketed by interglacial stages 13 and 15 which present high frequency oscillations with a relatively low $\delta^{18} \mathrm{O}$ variability. The morphology of these stages is rather typical and similar to that of the open ocean records (for instance, at DSDP Hole 552A in the Northeast Atlantic, Shackleton and Hall, 1984).

Identification of isotopic stages 17 through 21 is, in contrast, much more difficult and possibly the result of drilling disturbances in Core 6 (Sections 4, 5, and 6). As a result, the stage numbering that is proposed for this section is quite tentative (Fig. 3B).

\section{The Lower Pleistocene (53-84.4 mbsf, 0.8-1.66 Ma)}

Below stage 22, identification of isotopic stages is particularly difficult. Until very recently, the lack of high resolution isotopic records prevented the extension of isotope stage numbering into the early Pleistocene. The recent studies of Ruddiman et al. (1986) and Williams et al. (1988), however, have provided two different approaches to the problem. Williams and coauthors have assigned the stage boundaries $6 / 7(0.194 \mathrm{Ma})$ through $62 / 63$ (approximately $1.88 \mathrm{Ma}$ ) ages determined using linear interpolation between well dated biostratigraphic, paleomagnetic, and isotopic horizons. These authors have also proposed an interpolated composite $\delta^{18} \mathrm{O}$ record for the last 1.88 Ma. This approach differs from that of Ruddiman and coau- thors who named isotope stages 23-63 for the time period between 0.82 and $1.64 \mathrm{Ma}$, by "tuning" their isotope stage chronology to fit the 41,000 -yr obliquity periodicity. A tentative numbering for the period $0.8-1.7 \mathrm{Ma}$ with stages $24-58$ is proposed (Fig. 3C). Some stages may not be recorded due to the broad sampling interval or to sedimentary hiatuses. More high resolution and high quality data are needed before comparison can be made between this part of the Mediterranean isotopic record and the Atlantic ones.

\section{THE PLIOCENE-PLEISTOCENE BOUNDARY}

The INQUA subcommission on the Pliocene-Pleistocene boundary has recently proposed that the top of layer " $\mathrm{e}$ " in the Vrica section (southern Italy) should be used for definition of the Pliocene-Pleistocene boundary. This layer, which is located between the last occurrence of Discoaster brouweri, near 1.87 $\mathrm{Ma}$ (bottom of the Olduvai subchron) and the first appearance of $G$. oceanica s.l., near $1.56 \mathrm{Ma}$ (immediately above the Olduvai subchron) has been dated by interpolation at $1.64-1.66 \mathrm{Ma}$. An age of $1.66 \mathrm{Ma}$ has been proposed by Rio et al. (in press). This age also coincides with the Matuyama/Olduvai reversal boundary (Berggren et al., 1985). An interpolated age of 1.66 $\mathrm{Ma}$ is located at a depth of $86 \mathrm{mbsf}$ in Hole $653 \mathrm{~A}$ and not 84.4 mbsf as reported in Kastens et al. (1987) (Fig. 3C).

\section{THE PLIOCENE (86-215 mbsf, 1.66-4.6 Ma)}

The identification of isotopic stages in the Pliocene time is not easy as there does not exist yet a standard numbering scheme. An important increase in maximal and average $\delta^{18} \mathrm{O}$ values occurs between 122 and $115 \mathrm{mbsf}$ for $G$. bulloides and between 130 and $115 \mathrm{mbsf}$ for $G$. ruber. Maximal $\delta^{18} \mathrm{O}$ values increase from $+1.5 \%$ to $+2.5 \%$ for $G$. bulloides and from $0 \% 0$ to $+1 \%$ for G. ruber (Fig. 4). This suggests an important climatic change which may be related to the late Pliocene Northern Hemisphere ice build-up, near $2.4 \mathrm{Ma}$. This is supported by the Last Occurrence Datum (LAD) of Discoaster surculus near $111.49 \mathrm{mbsf}$, an event dated 2.4 Ma. Ages of the isotopic peaks 
Table 4. Oxygen and carbon isotope data for Site 654.

\begin{tabular}{|c|c|c|c|c|c|c|c|c|}
\hline $\begin{array}{l}\text { Levels } \\
\text { in the cores }\end{array}$ & $\begin{array}{l}\text { Depths } \\
\text { (mbsf) }\end{array}$ & $\begin{array}{l}\text { Ages } \\
1000 \mathrm{yr}\end{array}$ & $\begin{array}{c}\delta^{18} \mathrm{O} \% 0 \\
\text { G. bulloides }\end{array}$ & $\begin{array}{c}\delta^{13} \mathrm{C} \% 0 \\
G . \text { bulloides }\end{array}$ & $\begin{array}{l}\delta^{18} \mathrm{O} \% 0 \\
\text { G. falconensis }\end{array}$ & $\begin{array}{l}\delta^{13} \mathrm{C} \% 0 \\
\text { G. falconensis }\end{array}$ & $\begin{array}{l}\delta^{18} \mathrm{O} \%_{0} \\
G . \text { ruber }\end{array}$ & $\begin{array}{l}\delta^{13} \mathrm{C} \% 0 \\
\text { G. ruber }\end{array}$ \\
\hline $1-1-36$ & 0.36 & & & & & & 0.38 & 1.48 \\
\hline $1-1-92$ & 0.92 & & 3.15 & 0.21 & & & & \\
\hline $1-2-36$ & 1.86 & & 3.02 & -0.48 & & & & \\
\hline $1-2-92$ & 2.42 & & 3.62 & -0.67 & & & & \\
\hline $1-3-36$ & 3.36 & & 3.28 & -0.89 & & & & \\
\hline $1-3-92$ & 3.92 & & 3.73 & -0.72 & & & & \\
\hline $2-1-36$ & 4.96 & & 2.64 & -0.73 & & & & \\
\hline $2-1-92$ & 5.52 & & 3.23 & -0.73 & & & & \\
\hline $2-2-36$ & 6.46 & & 2.75 & -0.32 & & & & \\
\hline $2-3-36$ & 7.96 & & & & & & -1.01 & 0.65 \\
\hline $2-3-92$ & 8.52 & 215 & 0.90 & -0.89 & & & & \\
\hline $3-1-34$ & 12.34 & 278.2 & 2.6 & -0.46 & & & & \\
\hline $3-1-90$ & 12.90 & 289 & 3.37 & -0.64 & & & & \\
\hline $3-2-90$ & 14.40 & 314 & 1.85 & -0.43 & & & & \\
\hline $3-3-34$ & 15.34 & 327 & 3.23 & -0.01 & & & & \\
\hline $3-3-90$ & 15.90 & 334 & 2.82 & -0.84 & & & & \\
\hline $3-4-34$ & 16.84 & 347 & 3.23 & -0.01 & & & & \\
\hline $3-4-90$ & 17.40 & 355 & 2.24 & -0.43 & & & & \\
\hline $3-5-34$ & 18.34 & 368 & 2.10 & 0.27 & & & & \\
\hline $3-5-90$ & 18.90 & 377 & 1.69 & -0.36 & & & & \\
\hline $3-6-34$ & 19.84 & 392 & 1.53 & -0.87 & & & & \\
\hline $4-1-34$ & 21.74 & 423 & 3.03 & -1.07 & & & & \\
\hline $4-1-90$ & 22.30 & 436 & 2.28 & -1.00 & & & & \\
\hline $4-2-34$ & 23.24 & 459 & 3.67 & -0.06 & & & & \\
\hline $4-3-34$ & 24.74 & 478 & 2.91 & 0.11 & & & & \\
\hline $4-3-91$ & 25.31 & 486 & 2.51 & -0.04 & & & & \\
\hline $4-4-90$ & 26.84 & 510 & 1.98 & -0.37 & & & & \\
\hline $4-5-34$ & 27.74 & 524 & 2.38 & -0.85 & & & & \\
\hline $4-6-91$ & 28.30 & 558 & 2.67 & -1.03 & & & & \\
\hline $5-1-35$ & 31.15 & 734 & 2.03 & -0.58 & & & & \\
\hline $5-1-91$ & 31.71 & 769 & 2.52 & -0.45 & & & & \\
\hline $5-2-35$ & 32.65 & 827 & 2.36 & -0.59 & & & & \\
\hline $5-2-91$ & 33.21 & 861 & 3.29 & -1.28 & & & & \\
\hline $5-3-35$ & 34.5 & 919 & 1.55 & -1.28 & & & & \\
\hline $5-3-91$ & 34.71 & 950 & 2.39 & -0.58 & & & & \\
\hline $5-4-35$ & 35.65 & 958 & 2.16 & -0.41 & & & & \\
\hline $6-1-36$ & 40.66 & 997 & 1.84 & 1.03 & & & & \\
\hline $6-1-92$ & 41.22 & 1002 & 2.29 & -0.68 & & & & \\
\hline $6-2-36$ & 42.16 & 1009 & 2.09 & -0.86 & & & & \\
\hline $6-2-92$ & 42.72 & 1014 & 2.51 & -0.49 & & & & \\
\hline $6-3-36$ & 43.66 & 1021 & 1.63 & -0.66 & & & & \\
\hline $6-3-92$ & 44.22 & 1026 & 2.15 & -0.40 & & & & \\
\hline $6-4-36$ & 45.16 & 1033 & 1.89 & -0.50 & & & & \\
\hline $6-5-36$ & 46.66 & 1045 & & & & & 0.65 & 1.40 \\
\hline $6-5-92$ & 47.22 & 1050 & & & & & 0.75 & 0.76 \\
\hline $8-1-30$ & 59.40 & 1258 & 2.10 & 0.14 & & & 0.20 & 0.39 \\
\hline $8-2-32$ & 60.90 & 1298 & 2.23 & 0.05 & & & & \\
\hline $8-2-92$ & 61.50 & 1314 & 2.40 & -0.15 & & & & \\
\hline $8-3-52$ & 62.40 & 1331 & 2.85 & 0.19 & & & & \\
\hline $8-3-92$ & 63 & 1341 & 2.49 & -0.06 & & & & \\
\hline $8-4-32$ & 63.9 & 1356 & & -1.07 & & & & \\
\hline $8-4-92$ & 64.50 & 1366 & 1.02 & 0.06 & & & & \\
\hline $8-5-32$ & 65.40 & 1381 & 1.89 & 0.2 & & & & \\
\hline $8-5-92$ & 66 & 1391 & 2.65 & -0.02 & & & & \\
\hline $8-6-92$ & 66.90 & 1406 & 2.67 & 0.35 & & & & \\
\hline $9-1-36$ & 69.66 & 1452 & 2.87 & -0.03 & & & & \\
\hline $9-1-90$ & 70.20 & 1461 & 2.58 & -0.02 & & & & \\
\hline $9-2-36$ & 71.16 & 1477 & 1.98 & -0.48 & & & & \\
\hline $9-2-90$ & 71.70 & 1486 & 2.05 & -0.18 & & & & \\
\hline $9-3-36$ & 72.66 & 1514 & 1.96 & -0.10 & & & & \\
\hline $9-3-90$ & 73.20 & 1531 & 1.89 & -0.1 & & & & \\
\hline $10-1-36$ & 79.36 & 1706 & 1.98 & -0.21 & & & & \\
\hline $11-1-32$ & 89 & 1892 & & & & & 0.18 & 1.57 \\
\hline $11-2-38$ & 90.58 & 1912 & 1.76 & 0.52 & & & & \\
\hline $11-2-92$ & 91.12 & 1919 & 2.20 & 0.76 & & & & \\
\hline $11-3-32$ & 92.02 & 1931 & 2.07 & 0.49 & & & & \\
\hline $13-1-92$ & 108.92 & 2189 & 1.34 & 0.25 & 1.44 & -0.08 & & \\
\hline $13-3-92$ & 111.92 & 2270 & 0.97 & 0.25 & 1.27 & -0.08 & & \\
\hline $14-1-92$ & 118.62 & 2408 & 0.76 & -0.76 & & & & \\
\hline $14-2-32$ & 119.52 & 2423 & 1.35 & -0.32 & & & & \\
\hline $14-3-92$ & 121.62 & 2458 & 1.78 & -0.17 & & & & \\
\hline $14-4-32$ & 122.52 & 2472 & 1.49 & -0.14 & & & & \\
\hline $14-5-32$ & 123.02 & 2481 & 1.64 & 0.08 & & & & \\
\hline $15-1-92$ & 128.32 & 2576 & 1.31 & 0.15 & 1.61 & -0.16 & & \\
\hline $15-2-36$ & 129.22 & 2605 & 0.96 & 0.02 & 1.26 & -0.31 & & \\
\hline $15-2-92$ & 129.82 & 2625 & 1.27 & 0.22 & 1.57 & -0.11 & & \\
\hline $15-3-36$ & 130.76 & 2655 & 1.19 & 0.12 & 1.49 & -0.19 & & \\
\hline
\end{tabular}


Table 4 (continued).

\begin{tabular}{|c|c|c|c|c|c|c|c|c|}
\hline $\begin{array}{c}\text { Levels } \\
\text { in the cores }\end{array}$ & $\begin{array}{l}\text { Depths } \\
\text { (mbsf) }\end{array}$ & $\begin{array}{c}\text { Ages } \\
1000 \mathrm{yr}\end{array}$ & $\begin{array}{c}\delta^{18} \mathrm{O} \%_{0} \\
G . \text { bulloides }\end{array}$ & $\begin{array}{c}\delta^{13} \mathrm{C} \%_{0} \\
\text { G. bulloides }\end{array}$ & $\begin{array}{l}\delta^{18} \mathrm{O} \%_{0} \\
\text { G. falconensis }\end{array}$ & $\begin{array}{l}\delta^{13} \mathrm{C} \%_{0} \\
\text { G. falconensis }\end{array}$ & $\begin{array}{l}\delta^{18} \mathrm{O} \%_{0} \\
G . \text { ruber }\end{array}$ & $\begin{array}{l}\delta^{13} \mathrm{C} \%_{0} \\
G . \text { ruber }\end{array}$ \\
\hline $15-3-92$ & 131.32 & 2673 & 0.76 & 0.02 & 1.06 & -0.31 & & \\
\hline $15-4-35$ & 132.16 & 2700 & 0.65 & -0.42 & 0.95 & -0.75 & & \\
\hline $15-4-92$ & 132.82 & 2722 & 1.61 & 0.11 & & & & \\
\hline $15-6-36$ & 135.16 & 2797 & & & & & -1.71 & 0.84 \\
\hline $15-6-92$ & 135.72 & 2815 & & & & & -1.41 & 0.49 \\
\hline $15-7-39$ & 136.69 & 2846 & & & & & -0.11 & 1.44 \\
\hline $16-1-37$ & 137.27 & 2865 & 1.38 & 0.16 & & & & \\
\hline $16-2-37$ & 138.77 & 2914 & 0.80 & 0.03 & & & & \\
\hline $16-2-92$ & 139.32 & 2931 & 0.87 & -0.20 & & & & \\
\hline $16-3-37$ & 140.27 & 2962 & 1.22 & -0.20 & & & & \\
\hline $16-3-91$ & 140.82 & 2980 & 1.2 & -0.05 & & & & \\
\hline $16-4-37$ & 141.77 & 3010 & 1.11 & -0.33 & 1.68 & -0.34 & & \\
\hline $16-4-95$ & 142.35 & 3029 & 1.69 & -0.07 & & & & \\
\hline $16-5-37$ & 143.27 & 3059 & 1.11 & -0.32 & 1.41 & -0.65 & & \\
\hline $16-5-92$ & 143.82 & 3073 & 0.84 & -0.36 & 1.14 & -0.69 & & \\
\hline $16-6-37$ & 144.77 & 3090 & 1.15 & -0.19 & 1.45 & -0.52 & & \\
\hline $17-1-91$ & 147.31 & 3134 & 1.13 & 0.02 & 1.43 & -0.31 & & \\
\hline $17-3-35$ & 149.75 & 3176 & & & & & -0.73 & 1.40 \\
\hline $17-3-91$ & 150.31 & 3166 & 0.55 & 0 & 0.85 & -0.33 & & \\
\hline $17-4-35$ & 151.25 & 3202 & 1.02 & -0.24 & 1.32 & 0.07 & & \\
\hline $17-4-91$ & 151.81 & 3212 & 0.87 & -0.15 & 1.17 & 0.18 & & \\
\hline $18-2-36$ & 157.06 & 3303 & 1.11 & -0.19 & 1.41 & 0.14 & & \\
\hline $18-2-92$ & 157.62 & 3313 & 0.68 & -0.3 & 0.98 & 0.03 & & \\
\hline $18-4-32$ & 160.02 & 3354 & & & & & -0.44 & 1.60 \\
\hline $18-4-80$ & 160.50 & 3363 & & & & & -0.22 & 1.23 \\
\hline $18-4-92$ & 160.62 & 3365 & & & & & -0.76 & 0.67 \\
\hline $18-5-32$ & 161.52 & 3390 & 1.30 & 0.42 & 1.09 & -0.26 & & \\
\hline $18-5-92$ & 162.12 & 3391 & 0.98 & 0.17 & 1.25 & -0.23 & & \\
\hline $18-6-32$ & 163.02 & 3406 & 0.92 & 0.72 & 1.54 & 0.13 & & \\
\hline $18-6-92$ & 163.62 & 3416 & 1.80 & 0.51 & 1.63 & 0.05 & & \\
\hline $19-1-32$ & 164.42 & 3429 & 1.35 & 0.05 & & & G. obliquus & G. obliquus \\
\hline $19-1-92$ & 165.02 & 3439 & 1.41 & 0.10 & & & & \\
\hline $19-2-32$ & 165.92 & 3454 & 1.59 & 0.15 & & & & \\
\hline $19-2-92$ & 166.52 & 3464 & 1.17 & 0.06 & & & & \\
\hline $19-2-92$ & 166.52 & 3464 & & & & & -0.71 & 1.14 \\
\hline $19-3-32$ & 167.42 & 3478 & & & & & -1.06 & 1.50 \\
\hline $19-3-92$ & 168.02 & 3488 & 0.64 & 0.21 & & & & \\
\hline $19-4-32$ & 168.92 & 3503 & 0.84 & 0.41 & & & & \\
\hline $19-6-32$ & 171.92 & 3567 & 1.35 & 0.05 & & & & \\
\hline $20-1-20$ & 173.9 & 3614 & & & & & -0.02 & 1.85 \\
\hline $20-1-92$ & 174.62 & 3632 & & & & & -0.79 & 1.92 \\
\hline $20-2-20$ & 175.4 & 3651 & 1.50 & -0.03 & & & -1.12 & 1.35 \\
\hline $20-2-92$ & 176.12 & 3668 & 1.22 & 0.56 & 0.92 & -1.08 & 0.32 & 1.49 \\
\hline $20-3-20$ & 176.9 & 3687 & & & & & 0.05 & 2.05 \\
\hline $20-3-92$ & 177.62 & 3704 & & & & & 0.41 & 1.55 \\
\hline $20-4-20$ & 178.4 & 3723 & & & & & -1.01 & 1.38 \\
\hline $20-5-20$ & 179.9 & 3840 & & & & & -0.85 & 2.11 \\
\hline $20-5-91$ & 180.61 & 3851 & & & & & -1.37 & 1.13 \\
\hline $21-1-36$ & 183.56 & 3900 & & & & & -1.01 & 1.19 \\
\hline $21-3-36$ & 186.56 & 3949 & 0.97 & -0.15 & 1.27 & -0.48 & -0.25 & 1.64 \\
\hline $21-3-92$ & 187.12 & 3958 & 0.82 & -0.61 & & & -0.35 & 1.61 \\
\hline $21-4-35$ & 188.05 & 3974 & 1.68 & 0.10 & 1.04 & -0.34 & & \\
\hline $21-4-92$ & 188.62 & 3983 & 1 & -0.11 & & & -1.34 & 1.69 \\
\hline $21-5-36$ & 189.56 & 3999 & 1.64 & 0.39 & & & & \\
\hline $21-5-92$ & 190.12 & 4008 & 1.61 & 0.36 & 1.69 & 0.12 & & \\
\hline $23-1-36$ & 202.76 & 4153 & & & & & -0.07 & 1.25 \\
\hline $23-1-92$ & 203.32 & 4156 & & & & & -0.8 & 1.24 \\
\hline $23-2-36$ & 204.26 & 4160 & & & & & -1.36 & 1.24 \\
\hline $23-3-36$ & 205.76 & 4184 & & & & & -1.59 & 1.38 \\
\hline $23-4-36$ & 207.26 & 4207 & & & & & -1.30 & 1.19 \\
\hline $23-4-92$ & 207.82 & 4216 & 1.62 & & 0.18 & & & \\
\hline $24-1-92$ & 212.82 & 4293 & -0.2 & 0.94 & & & & \\
\hline $24-2-32$ & 213.72 & 4307 & 1.48 & 0.17 & & & & \\
\hline $24-2-92$ & 214.32 & 4317 & & 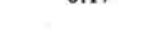 & & & -0.44 & 1.85 \\
\hline $24-3-32$ & 215.22 & 4331 & & & & & -0.28 & 1.75 \\
\hline 24-3-92 & 215.82 & 4340 & 1.17 & -0.34 & & & & \\
\hline $24-4-32$ & 216.72 & 4354 & 1.25 & -0.47 & & & & \\
\hline $24-4-92$ & 217.32 & 4363 & 1.40 & 0.17 & & & & \\
\hline $24-5-32$ & 218.22 & 4377 & 1.41 & -0.05 & & & -0.06 & 1.34 \\
\hline $24-5-92$ & 218.82 & 4387 & 1.57 & -0.59 & & & & \\
\hline $24-6-32$ & 219.72 & 4400 & 0.65 & -0.36 & & & & \\
\hline $25-1-36$ & 223.46 & 4458 & & & & & -0.47 & 1.59 \\
\hline 25-1-92 & 224.02 & 4467 & & & & & -0.56 & 1.47 \\
\hline $25-2-36$ & 224.92 & 4481 & & & & & -0.61 & 1.27 \\
\hline $25-2-92$ & 225.52 & 4490 & & & & & -0.53 & 1.35 \\
\hline $25-3-36$ & 226.46 & 4505 & & & & & -0.38 & 1.30 \\
\hline $25-3-92$ & 227.02 & 4513 & & & & & -0.09 & 1.21 \\
\hline
\end{tabular}


Table 4 (continued).

\begin{tabular}{|c|c|c|c|c|c|c|c|c|}
\hline $\begin{array}{l}\text { Levels } \\
\text { in the cores }\end{array}$ & $\begin{array}{l}\text { Depths } \\
\text { (mbsf) }\end{array}$ & $\begin{array}{l}\text { Ages } \\
1000 \mathrm{yr}\end{array}$ & $\begin{array}{c}\delta^{18} \mathrm{O} \% \\
\text { G. bulloides }\end{array}$ & $\begin{array}{c}\delta^{13} \mathrm{C} \%_{0} \\
\text { G. bulloides }\end{array}$ & $\begin{array}{c}\delta^{18} \mathrm{O} \%_{0} \\
\text { G. falconensis }\end{array}$ & $\begin{array}{l}\delta^{13} \mathrm{C} \% \\
\text { G. falconensis }\end{array}$ & $\begin{array}{l}\delta^{18} \mathrm{O} \%_{0} \\
G . \text { ruber }\end{array}$ & $\begin{array}{l}\delta^{13} \mathrm{C} \%_{0} \\
G . \text { ruber }\end{array}$ \\
\hline $25-4-92$ & 228.62 & 4537 & & & & & -1.51 & 1.58 \\
\hline $26-1-36$ & 233.06 & 4607 & & & & & -0.98 & 1.05 \\
\hline $26-1-92$ & 233.52 & 4616 & & & & & -0.31 & 1.14 \\
\hline $26-2-36$ & 234.56 & 4631 & & & & & -0.79 & 0.37 \\
\hline $26-2-92$ & 235.12 & 4640 & & & & & 0.03 & 0.96 \\
\hline $26-3-36$ & 236.06 & 4654 & & & & & 0.16 & 1.05 \\
\hline $26-3-92$ & 236.62 & 4664 & & & & & 0.86 & 1.43 \\
\hline $26-4-36$ & 237.56 & 4682 & & & & & -1.16 & 1.49 \\
\hline $26-4-92$ & 238.12 & 4693 & & & & & -0.76 & 1.86 \\
\hline $26-5-36$ & 239.06 & 4712 & & & & & -0.58 & 1.43 \\
\hline $26-5-92$ & 239.62 & 4723 & & & & & -0.63 & 1.61 \\
\hline
\end{tabular}

have been interpolated from those corresponding to the major faunal datums listed in Table 5 . The zonation is that reported in Kastens et al. (1987):

MP16: base at $12-1,19 \mathrm{~cm}$ (98.4 mbsf)

MPl5: base at $16-1,15 \mathrm{~cm}$ (136.2 mbsf)

MP14: base at $17-6,15 \mathrm{~cm}$ (153.3 mbsf)

MP13: base at $21-3,15 \mathrm{~cm}$ (186.3 mbsf)

MPl2: base at $23-5,75 \mathrm{~cm}(208.8 \mathrm{mbsf})$

In Figure 5 the oxygen isotopic data have been reported against the time scale defined above.

\section{THE PLIOCENE-PLEISTOCENE OXYGEN ISOTOPIC RECORD AT SITE 654}

The oxygen isotopic record obtained at Site 654 is poorly constrained both because of too large a sample interval and because of often indistinguishable reworked specimens. Therefore, the chronology proposed for this site is based essentially on paleomagnetic data of Channell and Torri (this volume) as well as biostratigraphically dated horizons or interpolated ages of major faunal changes also identified in Hole $653 \mathrm{~A}$ by Glaçon (Glaçon and Vergnaud Grazzini, this volume). The change in average $\delta^{18} \mathrm{O}$ values between the Pliocene period, before $2.4 \mathrm{Ma}$, and the Pleistocene, above $1.6 \mathrm{Ma}$, is clearly evident (Fig. 6).

\section{THE PLIOCENE-PLEISTOCENE CLIMATIC CHANGES}

At Hole 653A, the oxygen isotopic record of the G. ruber group presents the best resolution from approximately $210 \mathrm{mbsf}$ up to $75 \mathrm{mbsf}$, while the record of the $G$. bulloides group presents the best resolution from approximately $155 \mathrm{mbsf}$ up to the top of the hole. At Site 654, the resolution of the data is poor although some general trends are revealed by the $G$. bulloides record. Therefore, the discussion on the Pleistocene section will essentially bear on $G$. bulloides data of Hole 653A, while the interpretation of the Pliocene record will take into account the two species data at both Sites 653 and 654 . Our interpretations are based on examination and evaluation of the following parameters: (1) Maximal $\delta^{18} \mathrm{O}$ values of the glacial peaks, (2) Average $\delta^{18} \mathrm{O}$ values of the fluctuations (c), (3) Amplitude of the fluctuations of the $\delta^{18} \mathrm{O}$ values $(\mathrm{s}=$ variability).

Based on these criteria, five distinct intervals in the record of Hole 653A were identified. Four intervals correspond to rather steady values for the positive $\delta^{18} \mathrm{O}$ peaks of $G$. bulloides and $G$. ruber as well, and distinct average $\delta^{18} \mathrm{O}$ values of the fluctuations: Interval $\mathrm{V}$ from the base of the record up to $122 \mathrm{mbsf}$ $(\sim 2.7 \mathrm{Ma})$ for $G$. bulloides and from the base of the record up to 140 mbsf for $G$. ruber $(\sim 3.1 \mathrm{Ma})$; Interval III from 84.5 to 59 mbsf ( $\sim 1.55$ to $0.95 \mathrm{Ma})$; Interval II from 59 up to $21 \mathrm{mbsf}$ $(\sim 0.95$ to $0.34 \mathrm{Ma})$ and Interval I from $21 \mathrm{mbsf}$ to the top
( $\sim 0.34 \mathrm{Ma}$ to the recent time). Interval IV, which lasts from 3.1 to $1.55 \mathrm{Ma}$ for $G$. ruber and from 2.7 to $1.55 \mathrm{Ma}$ for $G$. bulloides is a transitional phase marked by a progressive increase in the average and maximal $\delta^{18} \mathrm{O}$ values of the fluctuations.

Increases in the variability of the $\delta^{18} \mathrm{O}$ signal occur at two different times in the sequence: initially around $0.95 \mathrm{Ma}$ when the amplitude of the fluctuations increases from $1.5 \%$ before, to $2 \% 0$ after; and a second time, around $0.4 \mathrm{Ma}$, when the amplitude of the oscillations becomes higher than 3\%0. At Site 654, interval $\mathrm{V}$, corresponding to maximal $\delta^{18} \mathrm{O}$ values of around $+1.8 \%$ for $G$. bulloides, can also be identified from the base of the record up to $118 \mathrm{mbsf}$ (around $2.4 \mathrm{Ma}$ ). Interval IV, between 118 and 75 mbsf (approximately 2.4 to $1.55 \mathrm{Ma}$ ) is less well documented but corresponds also to a trend of increasing $\delta^{18} \mathrm{O}$ values which leads to glacial $\delta^{18} \mathrm{O}$ values of around $+3 \%$. Interval III again is poorly documented but appears from 75 up to 35 mbsf (1.55 to $\sim 0.95 \mathrm{Ma})$. Interval II and I are difficult to separate. The top $35 \mathrm{~m}$ of the record corresponds to $\delta^{18} \mathrm{O}$ fluctuations of larger amplitude than below, with glacial $\delta^{18} \mathrm{O}$ values higher than $+3.5 \%$ and interglacial $\delta^{18} \mathrm{O}$ values reaching values similar to Pliocene ones at $8.5 \mathrm{mbsf}$.

\section{Interval V (4.6-3.1 Ma; the lower Pliocene; MPl2 to the top of MPl4)}

During this interval, the amplitude of the oxygen isotope fluctuations is elevated $(>1.5 \%)$ when compared to that recorded by $G$. bulloides in the Atlantic ocean at ODP Site 606 , at a latitude similar to that of Site 653 . At Site 606 the amplitude varies between $0.5 \% 0$ and $1 \%$ (Keigwin, 1987). Thus the variability observed at Hole $653 \mathrm{~A}$ cannot be attributed to an ice volume change. If the $1 \%$ excess in the magnitude of the fluctuations at Site 653 could be attributed to a temperature effect, it would mean that temperature fluctuations around $5^{\circ} \mathrm{C}$ (similar to those of the late Pleistocene time) already existed in the early Pliocene. This seems highly improbable, and we suggest that the large amplitude $\delta^{18} \mathrm{O}$ fluctuations recorded in the early Pliocene time of the Tyrrhenian basin are rather the result of cyclic changes in the water budget (Evaporation/Precipitation + River run-off Ratio $=\mathrm{E} / \mathrm{P}$ ). Additionally, during this interval, maximal $\delta^{18} \mathrm{O}$ values recorded by $G$. bulloides (around $+1.5 \%$ ) are higher than those recorded at site 606 (around $+0.85 \%$ ). Because we cannot argue that sea surface temperatures were cooler in the Mediterranean than in the Atlantic, we attribute this difference to a dryness effect. Pollen diagrams have shown that dryness was a characteristic of the Mediterranean area as early as the earliest Miocene and that a seasonal hydric contrast already existed at $25 \mathrm{Ma}$ (Pons et al., in press). A seasonal thermal contrast was gradually superimposed on the hydric contrast before 3.1 Ma. The dry season, however, was not really drastic until the seasonal thermal contrast was well established. The high amplitude $\delta^{18} \mathrm{O}$ fluctuations recorded before $3.1 \mathrm{Ma}$ in the 


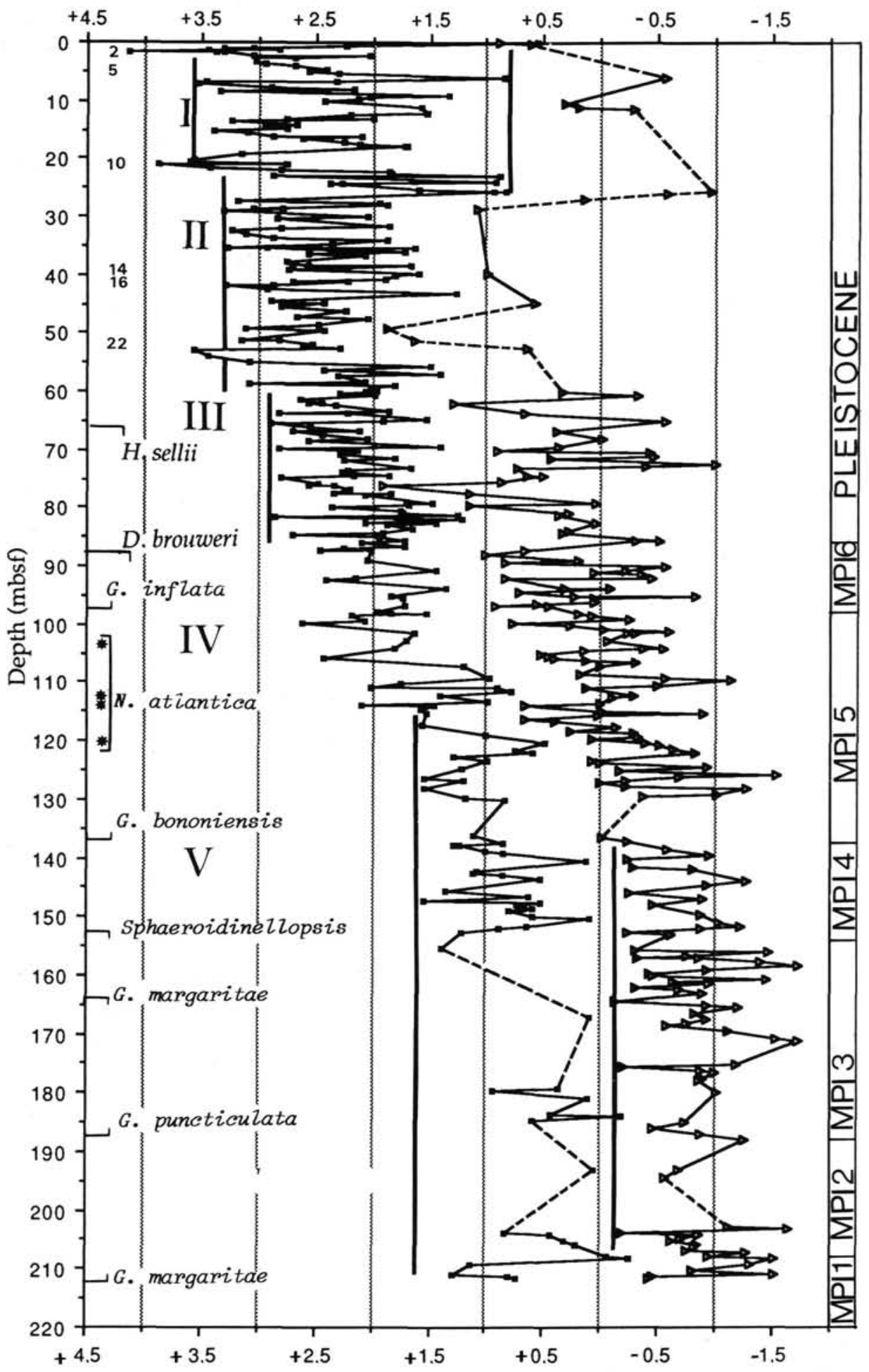

Figure 2. The $\delta^{18} \mathrm{O}$ records of $G$. bulloides (and G. falconensis) and G. ruber (and G. elongatus, G. obliquus, or $G$. obliquus extremus at ODP Hole 653A reported against depth below seafloor (in meters) with indication of Intervals I-V, as defined in the text. Major biostratigraphical events are indicated. $-=$ appearance; $\neg=$ extinction; ${ }^{*}=$ presence; $\Delta=G$. obliquus; $\mathbf{E}=G$. bulloides. 
Table 5. The various control points (isotopic stratigraphy and biostratigraphy) used for the reconstruction of the Pliocene-Pleistocene time scale at Hole 653A and Site 654. Isotopic terminations and stages after Martinson et al. (1987), SPECMAP (Imbrie et al., 1984), and typical faunistic changes identified by Glaçon (Glaçon and Vergnaud Grazzini, this volume). Faunal events after Rio et al. (this volume) and Sprovieri et al. (this volume).

\begin{tabular}{|c|c|c|c|}
\hline $\begin{array}{l}\text { Isotopic peaks } \\
\text { of transition } \\
\text { or peaks }\end{array}$ & $\begin{array}{c}\text { Age } \\
\text { (k.y.) }\end{array}$ & $\begin{array}{l}\text { Depth in } \\
\text { Hole 653A } \\
\text { (mbsf) }\end{array}$ & $\begin{array}{c}\text { Depth at } \\
\text { Site } 654 \\
\text { (mbsf) }\end{array}$ \\
\hline 1.1 & 8.5 & 0 & \\
\hline 2.0 & 12.1 & 1.1 & \\
\hline 2.2 & 17.8 & 1.5 & \\
\hline 2.23 & 23.2 & 2.4 & \\
\hline 5.5 & 123.8 & 6 & \\
\hline 6.0 & 129.8 & 6.5 & \\
\hline 6.2 & 135.1 & 7 & \\
\hline $6 / 7$ & 186 & 8.8 & \\
\hline 7.1 & 200 & 9.2 & \\
\hline 7.3 & 215 & 11 & ${ }^{\mathrm{a}} 8.52$ \\
\hline $7 / 8$ & 245 & 12.5 & ${ }^{a} 10.7$ \\
\hline 8.1 & 249.8 & 13.25 & \\
\hline 8.3 & 258.4 & 14.2 & \\
\hline 8.4 & 265.7 & 15 & \\
\hline $8 / 9$ & 303 & 16.5 & ${ }^{a_{13.6}}$ \\
\hline $9 / 10$ & 339 & 19.7 & \\
\hline $10 / 11$ & 362 & 22 & $\mathrm{a}_{17.99}$ \\
\hline $11 / 12$ & 423 & 27 & $a_{21.74}$ \\
\hline $12 / 13$ & 478 & 33.7 & a24.74 \\
\hline $13 / 14$ & 524 & 35.3 & ${ }^{a} 27.74$ \\
\hline $14 / 15$ & 565 & 38.5 & \\
\hline $15 / 16$ & 620 & 41 & \\
\hline $16 / 17$ & 659 & 42.6 & \\
\hline $17 / 18$ & 689 & 44.2 & \\
\hline $18 / 19$ & 726 & 46 & \\
\hline $19 / 20$ & 736 & 48.6 & \\
\hline \multicolumn{4}{|l|}{ Microfossil and nannofossil datums } \\
\hline P. lacunosa LAD & 460 & 29.12 & 23.28 \\
\hline Top "small Gephyrocapsa" & 950 & 58.92 & 34.64 \\
\hline H. sellii LAD & 1100 & 65.42 & 53.50 \\
\hline Gephyrocapsa spp. $>5.5 \mu \mathrm{m}$ & 1320 & 73.8 & 61.70 \\
\hline C. macintyrei LAD & 1490 & 80.27 & 71.90 \\
\hline Gephyrocapsa oceanica s.I.FAD & 1620 & 85.5 & 76 \\
\hline D. brouweri LAD & 1830 & 88.7 & 84.20 \\
\hline$G$. inflata $\mathrm{FOD}$ & 2100 & 98.5 & 105 \\
\hline D. surculus LAD & 2400 & 111.49 & 118.10 \\
\hline D. tamalis LAD & $2560 / 2600$ & 119.22 & 127.8 \\
\hline G. bononiensis FOD & 3070 & 135.8 & 143.6 \\
\hline End $D$. pentaradiatus paracme & 3270 & 146.4 & \\
\hline Sphenolithus spp. LAD & $3410 / 3360$ & 152 & 163.2 \\
\hline$R$. pseudoumbilicatus LAD & $3520 / 3550$ & 156 & 169.95 \\
\hline Beginning D. pentaradiatus paracme & 3550 & 158 & \\
\hline P. lacunosa FAD & 3740 & 165.6 & 179.1 \\
\hline A. delicatus LAD & 3750 & 166.3 & \\
\hline D. asymmetricus $\mathrm{FCO}$ & $3840 / 3830$ & 169.9 & 179.9 \\
\hline Amaurolithus spp. LCO & 4130 & 186.17 & \\
\hline G. puncticulata FOD & 4130 & 186.3 & 197.5 \\
\hline$H$. sellii FAD & 4160 & 187.67 & 204.2 \\
\hline G. margaritae FOD & 4660 & 208.8 & 236.4 \\
\hline
\end{tabular}

a Typical faunistic change identified by Glaçon.

Tyrrhenian basin may be partly accounted for by the gradual onset of a cooler season and the conformity of the dry season and the newly appeared warmer season.

\section{Interval IV (3.1-1.55 Ma; from the middle Pliocene (MPI5, MPl6) to the early Pleistocene)}

This interval is characterized by a gradual increase in the average $\delta^{18} \mathrm{O}$ values of the fluctuations as well as the maximal $\delta^{18} \mathrm{O}$ values. Values start to increase near $3.1 \mathrm{Ma}$ for $G$. ruber and near $2.7 \mathrm{Ma}$ for $G$. bulloides. The diachroneity in the two records may simply be due to a broad sampling interval in conjunction with poor recovery in Core 15 . Two phases can be iden- tified inside Interval IV. In the first phase, from approximately 3.1 (or $2.7 \mathrm{Ma}$ for $G$. bulloides) to $2.5 \mathrm{Ma}$, the increase in the $\delta^{18} \mathrm{O}$ values is rather rapid and averages $0.9 \%$. In the second phase, from 2.5 to approximately $1.55 \mathrm{Ma}$, the increase is more progressive and averages $0.5 \%$ for $\delta^{18} \mathrm{O}$ peak values.

\section{The 3.1 Ma Horizon: a Climatic Step in the Mediterranean} Basin?

A number of authors (Hodell et al., 1983; Leonard et al., 1983; Prell 1984, among others) have suggested that the first increase in $\delta^{18} \mathrm{O}$ values at $3.1 \mathrm{Ma}$ was essentially due to a climatic cooling and not to a significant growth of continental ice. Keigwin (1987), however, suggests that the increase indicates minor glaciations as well as bottom water cooling.

Because our records at Hole 653 A are only from planktonic foraminifers, temperature effects, as well as salinity effects related to the modifications of the $\mathrm{E} / \mathrm{P}$ ratio, are not easy to separate from ice volume effects. As recorded above, the $\delta^{18} \mathrm{O}$ values start to increase around 3.1 Ma in the Tyrrhenian basin. The 3.1 Ma horizon at Hole 653A does not, however, correspond to a rapid shift toward higher values as it does in other records (as for example in the North Atlantic at Hole 552A; Shackleton and Hall 1984; or even in the Tyrrhenian basin, at Site 132; Thunell and Williams, 1983). There is, however, an important turnover of planktonic (Zachariasse and Spaak, 1983) and benthic (Sprovieri, 1978; Van der Zwaan, 1983) foraminifers around 3.1 $\mathrm{Ma}$ in the Mediterranean. At Hole 653A, subtropical associations decrease between 170 and 136 mbsf and are replaced by temperate (transitional) associations (Rio et al., this volume). This event also coincides with the reintroduction of $G$. bononiensis in the Mediterranean (at 138 mbsf in Hole 653A) which is thought to be tied to the expansion of the cool eastern boundary current in the North Atlantic. It is followed by the extinction of Globoquadrina and Sphaeroidinellopsis.

Palynological studies also indicate that around 3.1 Ma, there occurred a new decrease in temperatures which enhanced drier conditions although varying from place to place (Suc, 1984, 1985). Such climatic changes may account for an increasing trend in $\delta^{18} \mathrm{O}$ values starting around $3.1 \mathrm{Ma}$.

\section{From 2.7 to 2.2 Ma: a Higher Climatic Instability Also Characterized the Mediterranean Area}

There is a general agreement concerning the onset of late Pliocene Northern Hemisphere glaciations around $2.4 \mathrm{Ma}$, but there is still a debate concerning the timing of climatic changes. High resolution studies on North Atlantic (DSDP Hole 552A; Shackleton et al., 1984; Shackleton and Hall, 1984) seem to indicate a rather sudden and large increase in the $\delta^{18} \mathrm{O}$ values of benthic foraminifers at $2.4 \mathrm{Ma}$, while the studies in the equatorial Pacific (DSDP Hole 572C; Prell, 1985) show a shift toward heavier values as well as a change in the $\delta^{18} \mathrm{O}$ variability, between 2.9 and $2.4 \mathrm{Ma}$, with several large positive isotopic excursions at $2.4 \mathrm{Ma}$. Other high resolution studies in the North Atlantic suggest that there should not be a stepwise increase in the $\delta^{18} \mathrm{O}$ values. Loubere (at DSDP Site $548 ; 1988$ ) showed that the onset of glaciations at $2.4 \mathrm{Ma}$ was part of a progressive climatic deterioration rather than a sudden climatic event while Keigwin at DSDP Site 606 (1987) showed that events of ${ }^{18} \mathrm{O}$ enrichment of increasing magnitude occurred at $3.1,2.7,2.6$, and $2.4 \mathrm{Ma}$.

In the Tyrrhenian basin, the $\delta^{18} \mathrm{O}$ records of $G$. bulloides and G. obliquus are slightly different. Glacial $\delta^{18} \mathrm{O}$ values of $G$. bulloides increase by steps between $122.7 \mathrm{mbsf}$, near $2.67 \mathrm{Ma}$ (with average values of about $+1.52 \% 0$ ), and 113.7 mbsf, near 2.46 $\mathrm{Ma}$ (with average values of about $+2.45 \%$. In addition, interglacial $\delta^{18} \mathrm{O}$ values of $G$. bulloides also increase, but this increase is progressive, between $120.3 \mathrm{mbsf}$, near $2.63 \mathrm{Ma}$ (with average of $\delta^{18} \mathrm{O}$ values of about $0 \% 0$ ), and $93.55 \mathrm{mbsf}$, near 1.96 

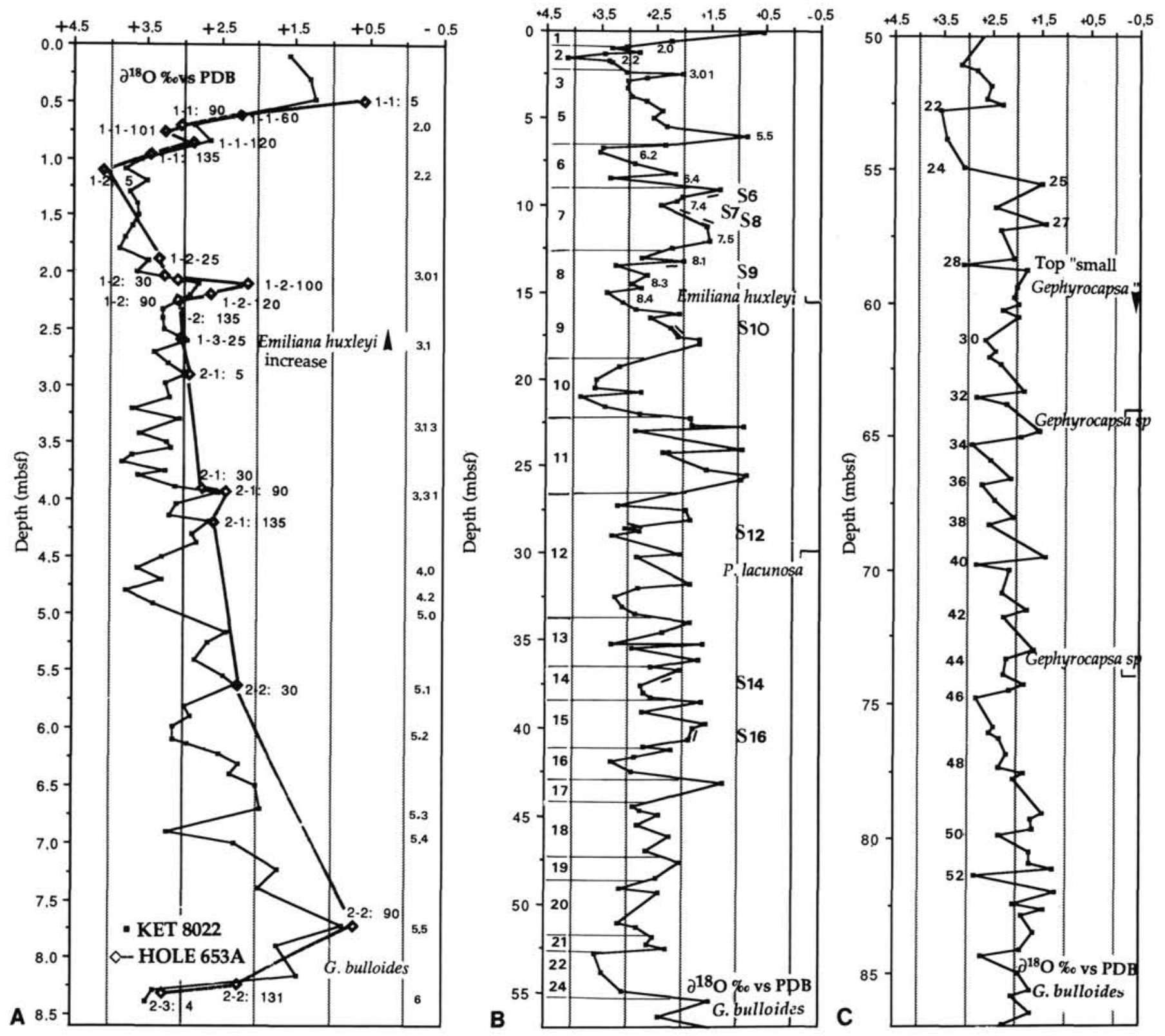

Figure 3. A. The late Pleistocene ${ }^{18} \mathrm{O}$ record of core KET 8022 (घ) (after Paterne et al., 1986). The location of the different samples of Hole 653A ( $\left.\diamond\right)$ on the basis of the $\delta^{18} \mathrm{O}$ values of $G$. bulloides is indicated. Stages and substages numbering is reported on the right side. B. The oxygen isotopic record of $G$. bulloides at Hole $653 \mathrm{~A}$ for the last $0.9 \mathrm{Ma}(57-0 \mathrm{mbsf})$. Sapropels are indicated (S) and are numbered following Ryan (1972). Isotope stage numbering is after Shackleton and Opdyke (1976) and Martinson et al. (1987). C. The oxygen isotopic record of G. bulloides at Hole 653A from the lower Pleistocene up to $0.9 \mathrm{Ma}(87-51 \mathrm{mbsf})$. A tentative numbering of isotope stages below stage 22 is proposed.

Ma (with average $\delta^{18} \mathrm{O}$ values of about $+1.52 \%$ ). It therefore appears that interglacial $\delta^{18} \mathrm{O}$ values of $G$. bulloides increase more than glacial values and in a more progressive scheme. Peaks of maximal $\delta^{18} \mathrm{O}$ values (glacial values) are recorded at four distinct levels: at $113.75 \mathrm{mbsf}(2.46 \mathrm{Ma}), 105.75 \mathrm{mbsf}(2.27$ $\mathrm{Ma}), 99.75 \mathrm{mbsf}(2.13 \mathrm{Ma})$, and $92.3 \mathrm{mbsf}$ (1.93 Ma). The apparent diachroneity with Site 606 record may partly be an artifact of interpolated ages that were calculated with the assumption that sedimentation rate does not vary between two dated horizons. Glacial $\delta^{18} \mathrm{O}$ values of $G$. obliquus display a rather rapid increase between $140 \mathrm{mbsf}(3.1 \mathrm{Ma})$ and $116 \mathrm{mbsf}(2.5$ Ma) with values increasing from $-0.25 \%$ at $3.1 \mathrm{Ma}$ to $+0.7 \%$ at $2.5 \mathrm{Ma}$. The overall increase of about $0.9 \%$ is therefore similar to that recorded by $G$. bulloides for glacial maxima of the same time interval.
Interglacial $\delta^{18} \mathrm{O}$ values of $G$. obliquus also increase but not in a progressive way from $-1.5 \%$ below $125 \mathrm{mbsf}(2.76 \mathrm{Ma})$ to $-0.5 \%$ above 105 mbsf $(2.25 \mathrm{Ma})$. The overall increase $(1 \% 0)$ is therefore less important than that recorded by $G$. bulloides $(1.5 \%)$ for interglacial minima. Thus, depending on the species analyzed, the record of the transition from preglacial to glacial Pliocene may be different. This transition appears to correspond to a more progressive ${ }^{18} \mathrm{O}$ enrichment between 3.1 and 2.5 Ma for the surface water species of the group $G$. ruber while it appears to occur more rapidly through several increasing steps between 2.67 and $2.46 \mathrm{Ma}$, for $G$. bulloides. This transition also corresponds to a similar increase of glacial $\delta^{18} \mathrm{O}$ values for the two groups of species while interglacial $\delta^{18} \mathrm{O}$ values increase less for the surface species of the $G$. ruber group than for $G$. bulloides. 
Table 6. Characteristic $\delta^{18} \mathrm{O}$ values of the late Pleistocene isotopic stages and substages of Core KET 8022 (after Paterne et al., 1986) and depth location of the same stages at Hole 653A.

\begin{tabular}{lcl}
\hline $\begin{array}{c}\text { Isotopic stages } \\
\text { and substages }\end{array}$ & $\begin{array}{c}\delta^{18} \mathrm{O} \text { values } \\
(\% \text { vs. PDB })\end{array}$ & \multicolumn{1}{c}{$\begin{array}{c}\text { Level at } \\
\text { Hole 653A }\end{array}$} \\
\hline Modern sediments & 1.5 & not recorded \\
2.0 & $\geq 2.8$ & $\begin{array}{l}1-1,101 \mathrm{~cm} \\
2.2\end{array}$ \\
$3.01-3.13$ & $\geq 3.9$ & $1-2,5 \mathrm{~cm}$ \\
3.31 & & $1-2,25 \mathrm{~cm}$ \\
5.0 & $\leq 2.1,30 \mathrm{~cm}$ \\
5.1 & 3.5 & $2-2,30 \mathrm{~cm}$ \\
4.0 & $\leq 2.0$ & not recorded \\
5.3 & $<1$ & $2-2,90 \mathrm{~cm}$ (adjusted value) \\
5.5 & &
\end{tabular}

One may therefore deduce that the period extending from 2.7 to $2.1 \mathrm{Ma}$ was a time of high climatic instability in the Mediterranean, recorded by a (relatively) important increase of glacial and interglacial $\delta^{18} \mathrm{O}$ values. It marks the transition between the preglacial Pliocene and the glacial Pliocene. Maximal $\delta^{18} \mathrm{O}$ values recorded by $G$. bulloides at the end of the transition (between 2.5 and $2.2 \mathrm{Ma}$ ) are around $2.46 \%$ in the Tyrrhenian basin while they average $1.5 \%$ in the Atlantic Site 606 .

Pollen diagrams reveal that temperatures decreased in Northern Europe, and Zagwin (1974) indicates a drastic cooling in the Netherlands and a Scandinavian glaciation during the lower Matuyama epoch, around $2.47 \mathrm{Ma}$, corresponding to the Pretiglian. This second step in boreal glaciation caused a further decrease in temperature and rainfall in Southern Europe (Cravatte and Suc, 1981; Suc, 1984, 1986; Combourieu Nebout, 1987). A seasonal thermal contrast was established with winter temperatures decreasing while a summer dryness appeared (Suc and Zagwin, 1983; Suc, 1984, 1986; Pons et al., in press). The increased seasonal contrast and the summer dryness extended to the whole Mediterranean area, even to the more humid zones including the Tyrrhenian basin. This may explain why the value of glacial $\delta^{18} \mathrm{O}$ maxima increased more in the Mediterranean than in the open ocean.

In addition, recent pollen analyses and oxygen isotope data from the Crotone series (southern Italy) (Combourieu Nebout, 1987) have revealed the occurrence of glacial cycles near $2.4 \mathrm{Ma}$. A very close relationship with the insolation changes (calculated for the month of July at $60^{\circ} \mathrm{N}$ latitude; Berger, in press) demonstrates that the Mediterranean also responded to orbitally forced climatic changes.

No major turnover in the planktonic foraminiferal fauna occurs in association with the climatic change at approximately $2.4 \mathrm{Ma}$, although it does correspond to the nearly complete disappearance of all Discoasters (Rio et al., this volume). In fact the interval 3.1 to $1.5 \mathrm{Ma}$ in the Tyrrhenian basin is characterized by the dominance of a temperate assemblage which gradually replaces the warm associations that became extinct near 3.1 $\mathrm{Ma}$ (Sprovieri et al., this volume). The reintroduction of the $G$. inflata group ( $G$. inflata) at 2.1 Ma corresponds to a short warming trend in the $\delta^{18} \mathrm{O}$ curve. This agrees with the assumption of Zachariasse and Spaak (1983) that a return to warmer climatic conditions was associated with a more eastward flowing eastern boundary current in the Atlantic.

\section{The Pliocene-Pleistocene Boundary and Interval III (1.55-0.95 Ma; the lower Pleistocene)}

The Pliocene-Pleistocene boundary does not correspond to any sudden change in the oxygen isotope ratio of $G$. bulloides (Figs. 3B and 5). However, this boundary corresponds to the initiation of a long term increase in $\delta^{18} \mathrm{O}$ values which culminates at $1.35 \mathrm{Ma}$ with glacial values of around $+2.8 \%$. A similar long term increase is also recorded in the type section of Vrica (southern Italy, Combourieu Nebout, 1987) where it ends around 1.3 Ma (Fig. 7). This long term trend is characterized by a low variability; it lasted about $200,000 \mathrm{yr}$ and has not been reported in open ocean sites. This suggests that between 1.5 and $1.3 \mathrm{Ma}$ in the Mediterranean, strong local overprints have obliterated the global ${ }^{18} \mathrm{O}$ signal. While surficial waters were getting cooler, the summer dryness may have been strong enough to obliterate the $\delta^{18} \mathrm{O}$ decreases induced by interglacial warming.

Traditionally, the Pliocene-Pleistocene boundary has been placed at the level where marine faunal elements, which at present are restricted to the boreal provinces, begin to occur in the Mediterranean (Italian) geological records. These elements are referred to as Boreal Guests (BG's) (Raffi, 1986). In fact the mollusk Arctica islandica as well as the benthic foraminifer $\mathrm{Hy}$ alinea balthica or the ostracode Cytheropteron testudo which were retained as Northern immigrants, are controlled by the changing parameters of the environment. Their occurrence during the Pleistocene has been gradual and discontinuous and does not represent a single event. However, the first appearance of Arctica islandica, which is proposed close to the top of the Olduvai subchron, slightly predates the long term $\delta^{18} \mathrm{O}$ increase mentioned above. Other evidence of climatic deterioration such as the coiling change in $N$. pachyderma are also reported near $84.4 \mathrm{mbsf}$ (near 1.59 Ma). Pollen diagrams show that the extinction of the Taxodiacae (Lona, 1962) occurred during the same period. Other palynological studies suggest a temperature fall between 1.7 and 1.5 Ma, documented by the expansion of Artemisia and the dominance of new taxa during the forest phases. To summarize, it appears that an important local evolution of the Mediterranean climate with a sharp fall in winter temperatures and a drop in rainfall may have obliterated the global climatic cycles recorded elsewhere in the open oceans.

During interval III, glacial $\delta^{18} \mathrm{O}$ values remain quite steady (near $2.8 \%$ ). The amplitude of the $\delta^{18} \mathrm{O}$ fluctuations is around $1.2 \%$, a value which is slightly lower than the amplitude of the preceding intervals. This suggests that during this interval, there may not have been any drastic changes in the $\mathrm{E} / \mathrm{P}$ ratio values, and the maximal extension of the polar ice caps did not vary much. The comparison of the record with that obtained at the Vrica section for the same time interval reveals the following facts: up to $1.3 \mathrm{Ma}$ at Hole $653 \mathrm{~A}$, interglacial $\delta^{18} \mathrm{O}$ values recorded by $G$. bulloides are generally higher than at Vrica. This suggests warmer and/or more humid interglacial stages in the central Mediterranean than in the west Mediterranean. After 1.3 $\mathrm{Ma}$, the $\delta^{18} \mathrm{O}$ values of $G$. bulloides remain permanently higher in the Tyrrhenian Site than in the Crotone section, suggesting a more important dryness or cooling effect in the Tyrrhenian ba$\sin$ (Fig. 7). This period follows an episode of tectonic readjustment in the Appennines. Related changes in the physiography of the basins may have brought about modifications in the water mass circulations and isolated distinct paleoceanographic provinces. This interval also corresponds to an increase in the terrigenous component of the sediment (Kastens et al. 1987).

\section{Interval II (0.95-0.34 Ma; the middle Pleistocene)}

Starting with isotopic stage 28 , higher glacial $\delta^{18} \mathrm{O}$ values are recorded. Near 60 mbsf, a sharp decrease in the abundance of planktonic foraminiferal species referred to as "warm temperate" (factor 3 of Sprovieri et al., this volume) is also recorded.

The base of this interval also marks an important change in the variability of all Pliocene-Pleistocene oxygen isotopic records. Different estimates have been proposed for the exact timing of this change. In the study of the Quaternary section of Hole 502B, Prell (1982) proposed that the change of mode occurred at about $0.73 \mathrm{Ma}$, beneath the Bruhnes/Matuyama bound- 


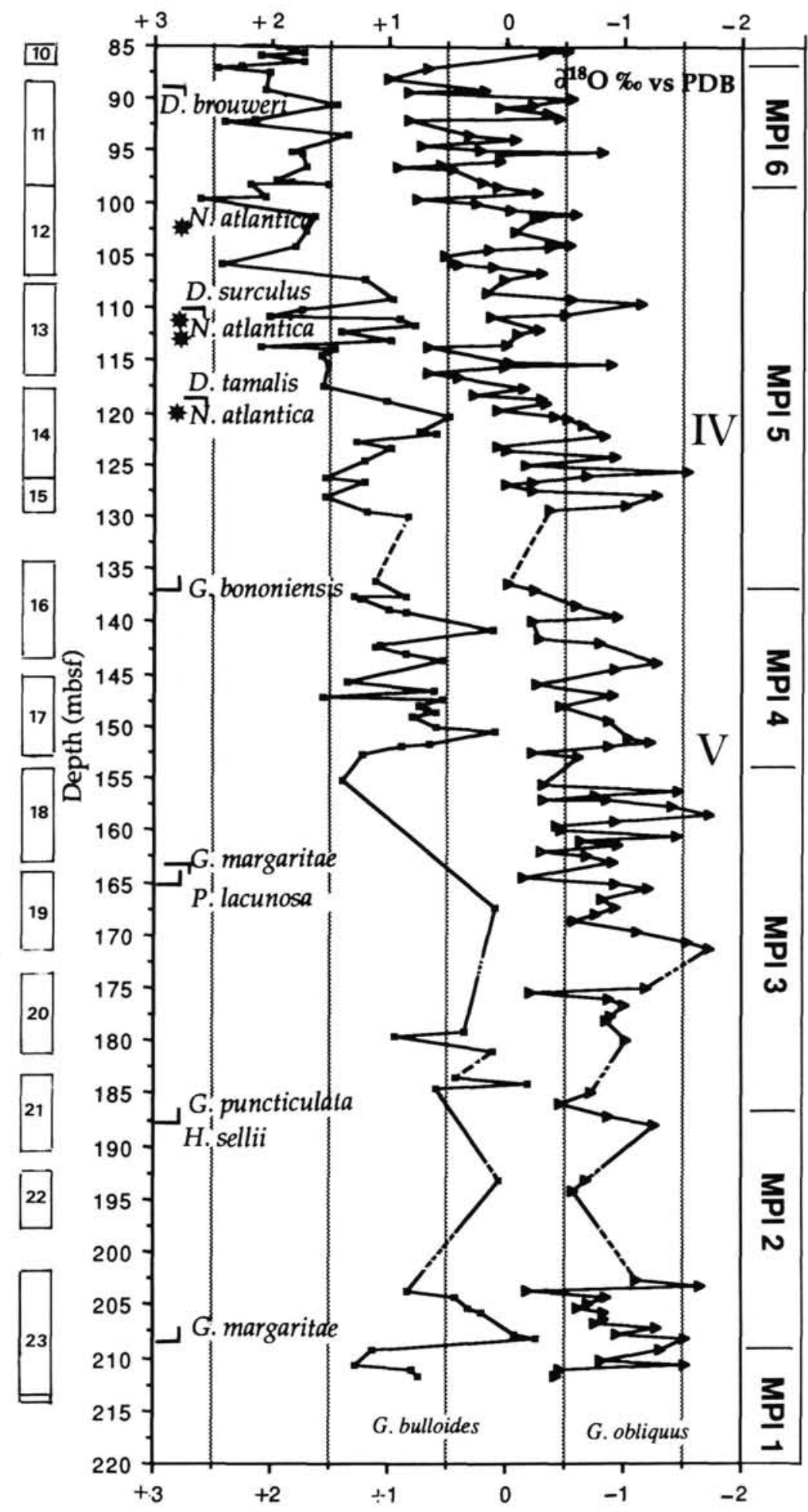

Figure 4. The Pliocene ${ }^{18} \mathrm{O}$ record of $G$. bulloides $(\square)$ and $G$. ruber $(\downarrow)$ (85-211 mbsf) at Hole 653A. Interval IV and V are indicated. The zonation is after Kastens, Mascle, et al. (1987). Major bioevents are also reported $-=$ appearance; $\neg$ = extinction; * $=$ presence. Core numbers are on the left side.

ary. Jansen and Sejrup (1987) placed this change at 0.9 Ma. Our results suggest that the boundary between the two modes be located near stage $28(\sim 58.5 \mathrm{mbsf})$ approximately dated $0.94 \mathrm{Ma}$, an age which is close to that proposed by the last authors. The amplitude of this middle Pleistocene change in the Mediterranean can be compared with those measured at other sites (Table 7). The major feature of this period, however, is the increase in the $\delta^{18} \mathrm{O}$ variability which is 1.5 times higher than that of the lower Pleistocene and Pliocene (the benthic record at ODP Site 610 is an exception). This suggests more severe climatic fluctuations related to larger polar front migrations in the North Atlantic. In addition the average $\delta^{18} \mathrm{O}$ values also increase, but the amplitude of this increase varies with 

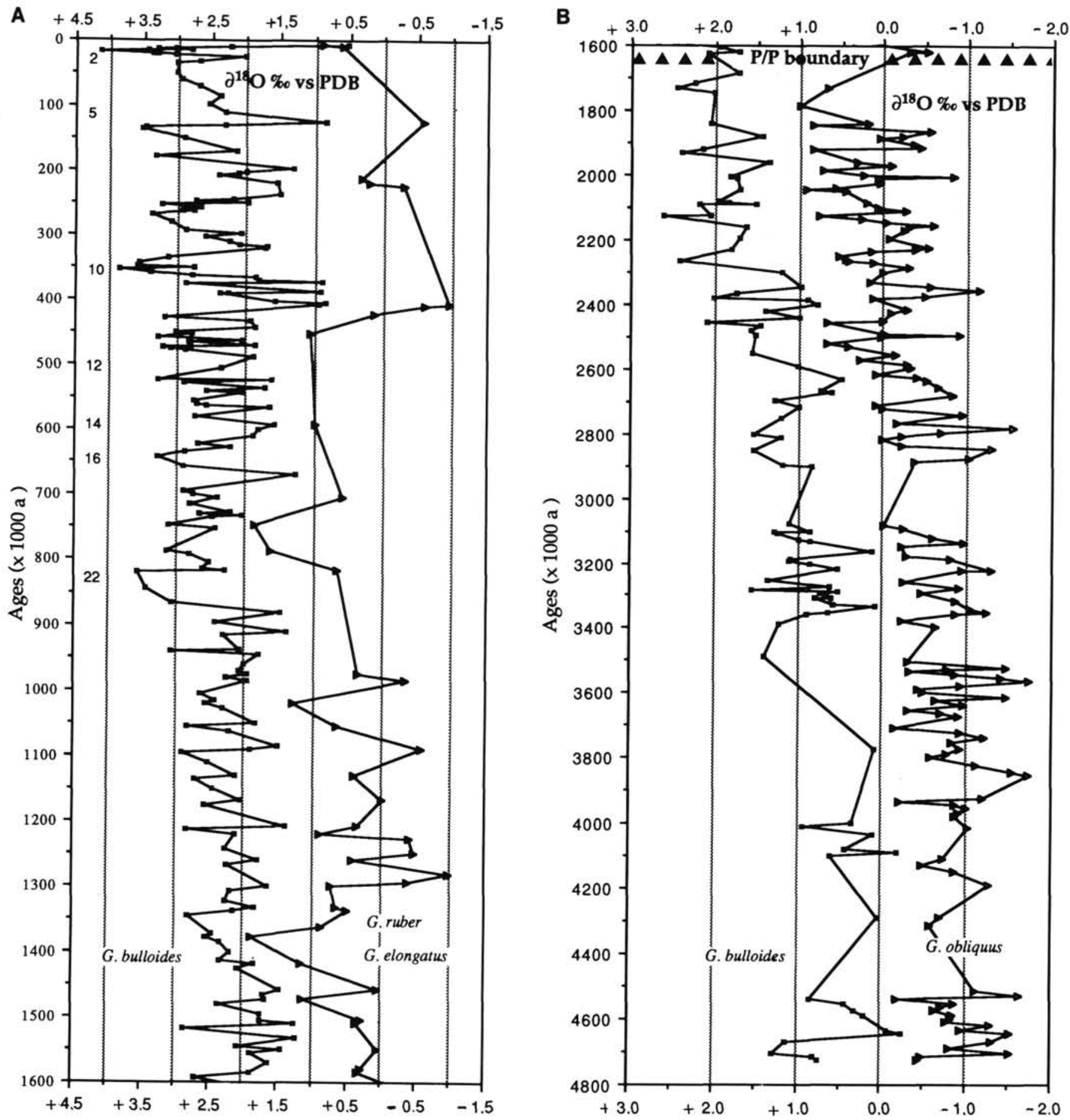

Figure 5. The ${ }^{18} \mathrm{O}$ record of $G$. bulloides and $G$. ruber as a function of interpolated dates. A. From $1.6 \mathrm{Ma}$ to the top of the record. B. From the base of the record to $1.6 \mathrm{Ma}$.

the sites. The smallest increase common to benthic and planktonic foraminifera is around $0.35 \%$ and may represent an increase in the volume of continental or Arctic ice cover. The higher increase recorded by $G$. bulloides at Hole $653 \mathrm{~A}$ may be related to an additional and local salinity and/or a cooling effect.

\section{Interval I (0.34 Ma to the top of the record)}

This interval is marked by an increase in the $\delta^{18} \mathrm{O}$ variability, which corresponds both to an increase in the $\delta^{18} \mathrm{O}$ glacial values and to a decrease in the interglacial $\delta^{18} \mathrm{O}$ values. This change in the variability can also be seen at other sites. Other ocean surface and terrestrial records demonstrate a major climate change in the midBrunhes $\left(4 \times 10^{5}\right.$ to $3 \times 10^{5} \mathrm{yr}$ ago) (Jansen et al., 1986). The changes should not be necessarily synchronous but fall into two distinct groups. In the Northern Hemisphere, north of $20^{\circ} \mathrm{N}$, records show a trend toward more "glacial" conditions. In contrast, the records from equatorial regions and the Southern Hemisphere suggest more "interglacial" conditions and higher sea surface tem- 


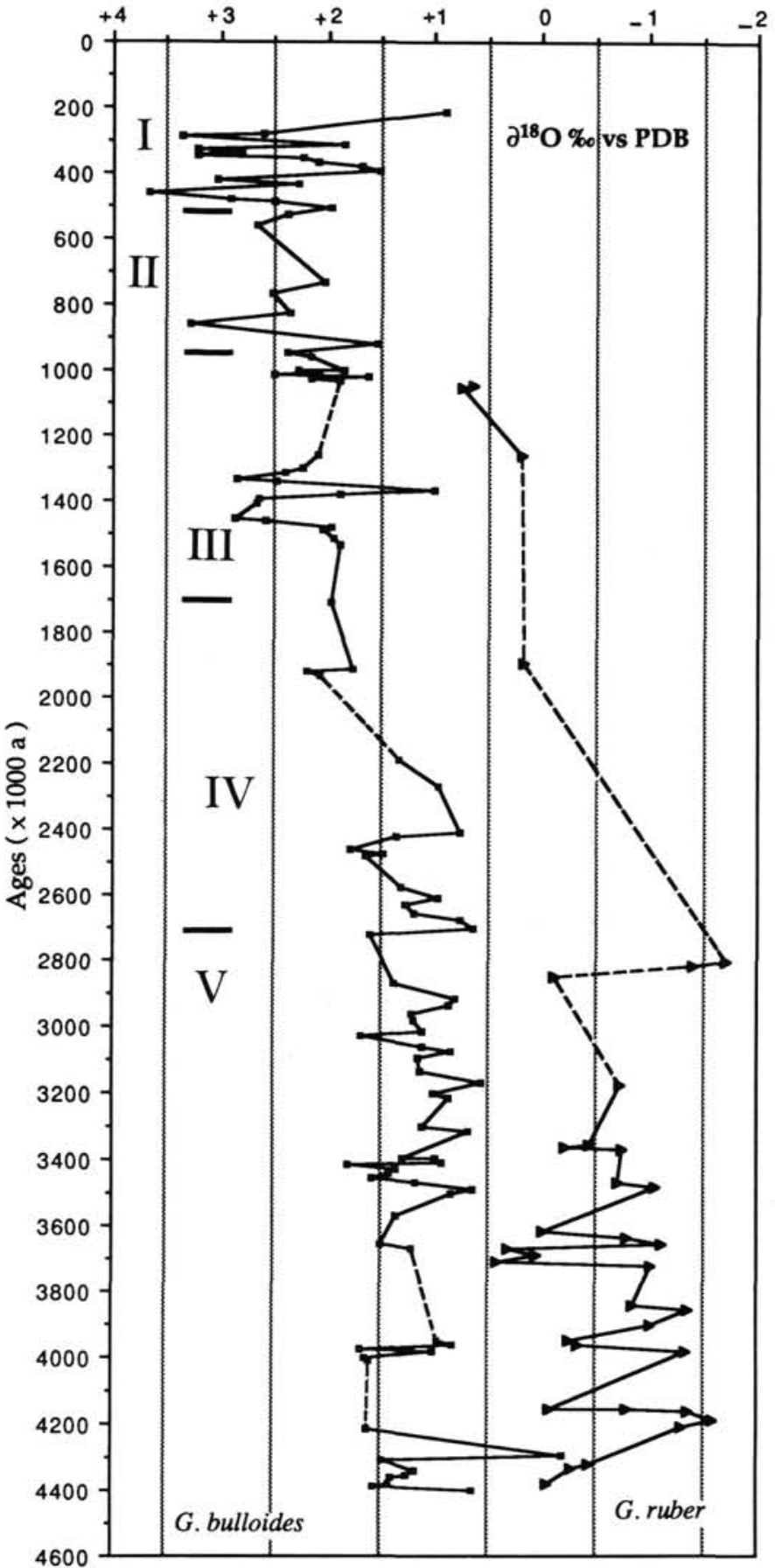

Figure 6. The Pliocene-Pleistocene ${ }^{18} \mathrm{O}$ records of $G$. bulloides $(\boldsymbol{\square})$ and G. ruber $(\triangleright)$ against interpolated ages at Site 654 . The transition between the preglacial Pliocene and the glacial Pliocene (2.7-1.9 Ma = Interval IV) is well apparent as well as the increase in the variability in the upper part of the record, above $0.5 \mathrm{Ma}$ (Interval I). Intervals I-V are indicated.

peratures (SST). Jansen et al. (1986) hypothesized perturbation by the orbital eccentricity cycle as a possible cause of this "mid Brunhes event."

In the Mediterranean the large $\delta^{18} \mathrm{O}$ fluctuations may result from large SST changes controlled by the increased continental seasonality. The last glacial-interglacial change in the $\delta^{18} \mathrm{O}$ values of $G$. bulloides is higher than $3.5 \%$. Causes of this abnormal amplitude

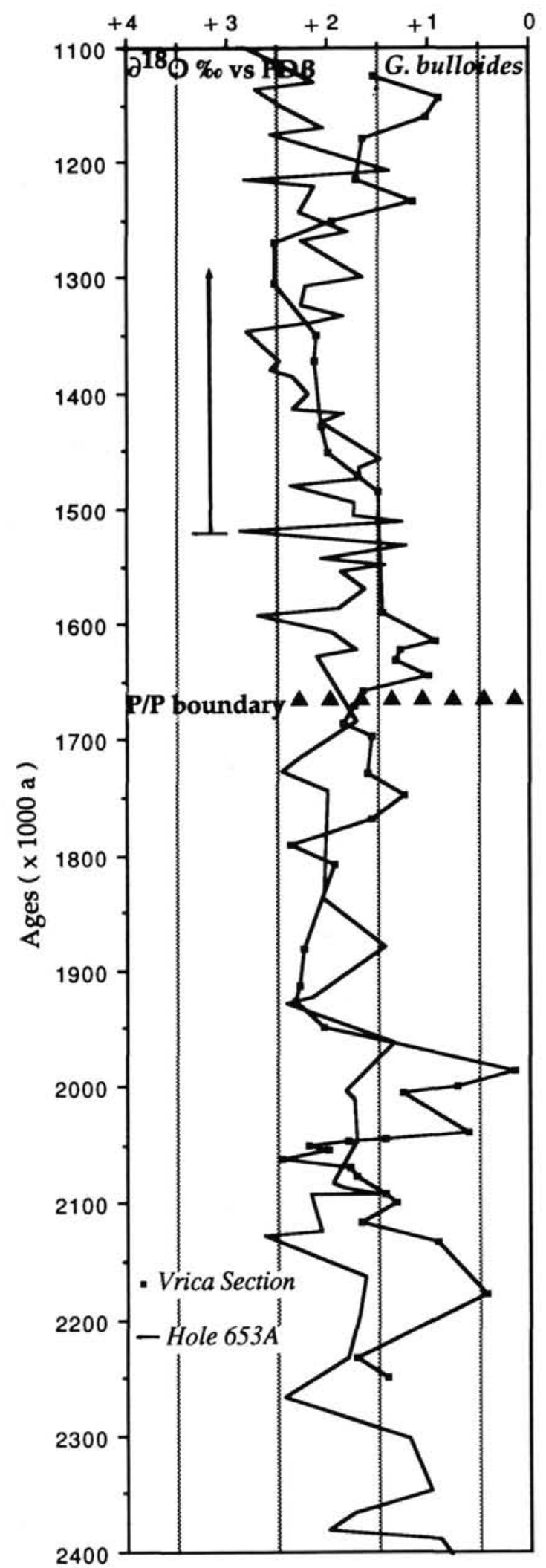

Figure 7. Comparison of the $\delta^{18} \mathrm{O}$ records of $G$. bulloides at the Vrica section (-) and at Hole 653A (-) for the time interval 1.1-2.4 Ma. The long increasing trend in $\delta^{18} \mathrm{O}$ values after $1.6 \mathrm{Ma}$ is indicated. 
Table 7. Compared changes in the $\delta^{18} O$ variability $(\sigma)$ in different oceans (and DSDP Sites) in the middle Pleistocene (near 0.8 Ma).

\begin{tabular}{|c|c|c|c|c|c|c|}
\hline \multirow[b]{2}{*}{$\begin{array}{l}\text { Period } \\
\text { (Ma) }\end{array}$} & \multirow{2}{*}{ 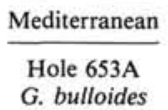 } & \multicolumn{3}{|c|}{ Atlantic } & \multicolumn{2}{|c|}{ Pacific } \\
\hline & & $\underset{\text { Benthic }}{\mathrm{H}}$ & le 610 & $\begin{array}{c}\text { Hole } 552 \mathrm{~A} \\
\text { Benthic }\end{array}$ & $\begin{array}{l}\text { Hole 502B } \\
\text { Planktonic }\end{array}$ & $\begin{array}{l}\text { V28-239 } \\
\text { Benthic }\end{array}$ \\
\hline $0-0.8$ & $\begin{array}{l}2.55 \\
0.67\end{array}$ & $\begin{array}{l}3.9 \\
0.47\end{array}$ & $\begin{array}{l}2.53 \\
0.98\end{array}$ & $\begin{array}{r}4.2 \\
\sim 1\end{array}$ & $\begin{array}{r}-0.46 \\
0.50\end{array}$ & $\begin{array}{r}-0.98 \times \\
0.35 \sigma\end{array}$ \\
\hline $0.8-2.5$ & $\begin{array}{l}1.99 \\
0.39\end{array}$ & $\begin{array}{l}3.5 \\
0.41\end{array}$ & $\begin{array}{l}1.57 \\
0.53\end{array}$ & $\begin{array}{l}3.75 \\
0.5\end{array}$ & $\begin{array}{r}-0.81 \\
0.28\end{array}$ & $\begin{array}{r}-1.01 \times \\
0.18 \sigma\end{array}$ \\
\hline$\Delta$ & $\begin{array}{l}0.55 \\
0.28\end{array}$ & $\begin{array}{l}0.40 \\
0.06\end{array}$ & $\begin{array}{l}0.96 \\
0.4\end{array}$ & $\begin{array}{l}0.46 \\
0.5\end{array}$ & $\begin{array}{l}0.35 \\
0.22\end{array}$ & $\begin{array}{l}0.07 \times \\
0.17 \sigma\end{array}$ \\
\hline
\end{tabular}

have been extensively discussed (Vergnaud Grazzini, 1973, 1975, 1977, 1983, 1985; Vergnaud Grazzini et al. 1986; Thunell et al., 1987). The global control of the $\delta^{18} \mathrm{O}$ signal by ice volume changes in the late Pleistocene is superimposed to both a dryness effect and a cooling of surficial (and deep) waters during glacial stages. This is corroborated by pollen studies of the upper Pleistocene stadial intervals that show a predominance of steppic and herbaceous vegetation. This later result suggests both arid and cold conditions (Pons et al., in press).

\section{CARBON ISOTOPE RECORDS}

The present day Mediterranean waters are characterized by a low surface to bottom $\Delta^{13} \mathrm{C}$ gradient, due to the fact that deep and intermediate waters produced in the Mediterranean carry the carbon isotopic signature of the surface waters. Due to a short residence time ( $\sim 100 \mathrm{yr}$, Lacombe et al., 1981) and a surface productivity which is very low, the carbon isotope composition of deep and intermediate waters is not altered significantly before the waters leave the Mediterranean. $\delta^{13} \mathrm{C}$ values for surficial waters are between 1.5 and $1.6 \%$. Intermediate waters $\mathrm{\Sigma CO}_{2}$ displays rather steady $\delta^{13} \mathrm{C}$ values which are around $1.1 \% 0$ at their source area in the east Mediterranean and decrease to $0.9 \%$ in the west Mediterranean. $\delta^{13} \mathrm{C}$ values of the deep $\mathrm{\Sigma CO}_{2}$ are around $1.2 \%$ near the source areas in the Gulf of Lion and south of the Adriatic Sea, and decrease to $1.0 \% 0$ away from these sources. The surface to deep $\delta^{13} \mathrm{C}$ gradient therefore averages $0.5 \%$ in the modern Mediterranean (Duplessy, 1972; Pierre et al., 1986).

In addition, the major component of Mediterranean surface waters is the low-nutrient North Atlantic surface water, with a rather high ${ }^{13} \mathrm{C}$ content $\left(\delta^{13} \mathrm{C}=2 \%\right)$ : the lower ${ }^{13} \mathrm{C}$ content which is observed nowadays in the surficial Mediterranean waters is not easy to explain.

There have been some attempts to reconstruct the past hydrography of the Mediterranean through the changes of the surface to deep $\delta^{13} \mathrm{C}$ gradient recorded by benthic and planktonic foraminiferal shells (Vergnaud Grazzini 1983; Thunell et al., 1987). In particular, for Thunell and coauthors, an estuarine type of flow existed between the Mediterranean and the Atlantic in the earliest Pliocene: waters entered the Mediterranean at depth and flowed out at the surface. Such conclusions were based on the observation of a higher-than-present-day surface to deep $\delta^{13} \mathrm{C}$ gradient at DSDP Site 132 in the Tyrrhenian basin; they were supported by ostracode data (Benson, 1975; Van Harten, 1984).

Tectonic readjustments as well as the climatic evolution toward drier (and cooler) conditions were responsible for the change in the water budget of the Mediterranean and the current reversal of its exchanges with the Atlantic. Some questions may arise, however, concerning the modality of this reversal. Circulation patterns inside the Mediterranean might have changed from upwelling to downwelling systems as we know them today. The implications of such changes for the ${ }^{13} \mathrm{C}$ distribution in the water column are not clear. Deep and intermediate water sources may not have been located at the same places as they are today and may not have been synchronous throughout the basin. A two layered system may have also preceded the three layered system of the present day Mediterranean.

The Pliocene-Pleistocene ${ }^{13} \mathrm{C}$ records of two planktonic foraminiferal species which are presently available at Sites 653 and 654 in the Tyrrhenian basin do not enable us to reconstruct the past changes in the Mediterranean hydrology. One may, however, try to discriminate the changes which are related to the global modifications of the $\mathrm{CO}_{2}$ balance from the local climatic and hydrographic evolutions. In the present day Mediterranean, the $\delta^{13} \mathrm{C}$ values of the surficial species $G$. ruber are between $+1.30 \%$ and $1.50 \%$ and therefore reflect quite well the $\delta^{13} \mathrm{C}$ values of the surficial $\Sigma \mathrm{CO}_{2}$ (Vergnaud Grazzini et al., 1986). Interpretation of the ${ }^{13} \mathrm{C}$ content of $G$. bulloides is more complex. Plankton tow observations show that maximal abundances of this species occur in spring and are dependent on a shallow regenerated production. A strong thermo-halocline creates a seasonal density barrier which allows for the surface nutrients to be regenerated at a shallow depth. Consequently, in the Mediterranean, $G$. bulloides is generally found near or at the first level of oxygen minimum. As a result, episodes of higher stratification in the late Pleistocene (e.g., those related to the first step of the last deglaciation or to the water stagnation in the east Mediterranean) correspond to a decrease in the ${ }^{13} \mathrm{C}$ content of this species. Although it does not precipitate its shell in equilibrium with the ambient $\Sigma \mathrm{CO}_{2}$ (its average $\delta^{13} \mathrm{C}$ value is, today, between $-0.6 \%$ and $-0.7 \%$ ), the relative ${ }^{13} \mathrm{C}$ changes of $G$. bulloides may bring some information on the variations of the density stratification in the upper water layers. The differences observed between the $\delta^{13} \mathrm{C}$ records of the two species at Hole $653 \mathrm{~A}$ suggest that different depth requirements are responsible for an overall range of $\delta^{13} \mathrm{C}$ fluctuations which is larger for $G$. bulloides $(2 \%)$ than for $G$. ruber $(1.5 \%)$.

Furthermore, examination of the ${ }^{13} \mathrm{C}$ records for the last 4.5 Ma (Fig. 8) shows that rather large fluctuations are displayed by the two species since the early Pliocene, pointing to rapid changes in the $\mathrm{CO}_{2}$ budget of surface waters (Fig. 8). Three main phases can be identified in the records. In a first phase, up to $\sim 1.5 \mathrm{Ma}$, the $\delta^{13} \mathrm{C}$ fluctuations occur above the minimal value of $-0.7 \%$ for $G$. bulloides and around a steady mean value of $+1.3 \%$ for $G$. ruber. From 1.5 to $0.5 \mathrm{Ma}$, mean and minimal $\delta^{13} \mathrm{C}$ values display a decreasing trend, the lowest values of the record being reached between 0.8 and $0.5 \mathrm{Ma}$. From $0.5 \mathrm{Ma}$ to the top of the record the $\delta^{13} \mathrm{C}$ values increase slightly and reach their present day level.

\section{The ${ }^{13} \mathrm{C}$ Record Before $1.5 \mathrm{Ma}$}

For the $G$. ruber group, episodes of higher ${ }^{13} \mathrm{C}$ content recorded before 1.5 Ma, may reflect an enhanced productivity of 

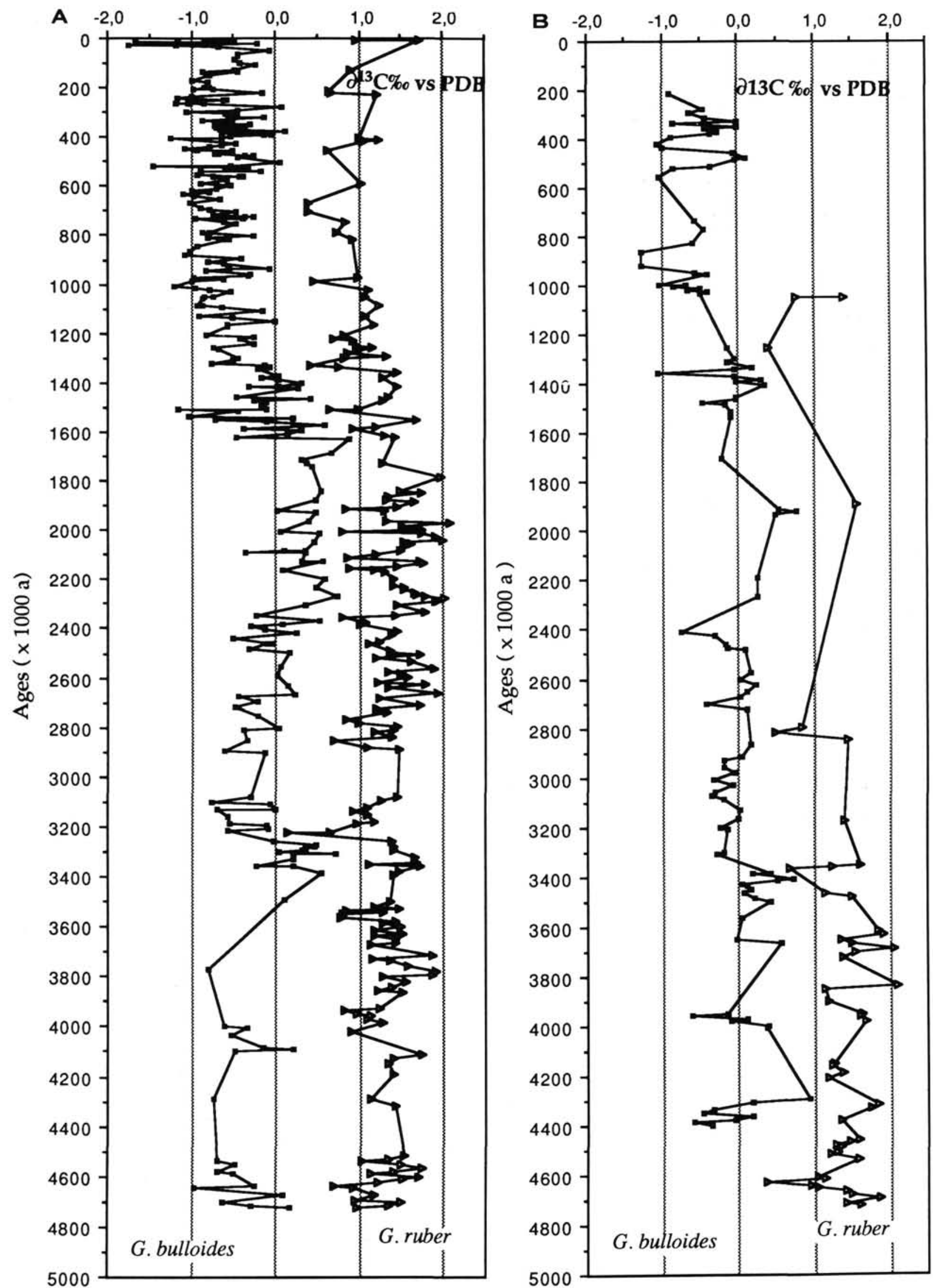

Figure 8. The Pliocene-Pleistocene $\delta^{13} \mathrm{C}$ records of $G$. bulloides $(\mathbf{D})$ and $G$. ruber $(\triangleright)$ at Hole $653 \mathrm{~A}(\mathbf{A})$ and $G$. bulloides at Site $654(\mathbf{B})$ as a function of interpolated dates. 
phytoplankton in surface waters. By this process, light isotopes become enriched in the phytoplankton, enriching surface $\mathrm{\Sigma CO}_{2}$ in ${ }^{13} \mathrm{C}$, which is reflected in surficial planktonic foraminiferal shells. When such episodes coincide with a high stratification of the waters, probably related to a strengthening of the thermohalocline, the $\delta^{13} \mathrm{C}$ values of $G$. ruber increase while the $\delta^{13} \mathrm{C}$ values of $G$. bulloides decrease. This figure corresponds to the intervals 4.6-4.05 $\mathrm{Ma}, 3.9-3.6 \mathrm{Ma}$, and 3.25-2.66 Ma. In contrast, when episodes of high primary production coincide with low stratified waters, the ${ }^{13} \mathrm{C}$ content of both species, $G$. ruber and $G$. bulloides increases simultaneously. This figure corresponds to the intervals 4.05-3.9 Ma, 3.6-3.25 Ma, 2.66-2.46 $\mathrm{Ma}, 2.27-1.98 \mathrm{Ma}, 1.92-1.65 \mathrm{Ma}$, and 1.47-1.36 Ma.. The interval 2.66-1.65 Ma corresponds to the transition between the preglacial Pliocene and the glacial Pliocene-Pleistocene. Episodes of severe cooling of the surficial waters and increasing dryness over the Mediterranean basin (with the related changes in the water budget) have been deduced from the $\delta^{18} \mathrm{O}$ records. In addition, the paleoceanography during this interval was characterized by long periods of intensified vertical mixing. This figure heralds what may be deduced from the late Pleistocene Mediterranean ${ }^{13} \mathrm{C}$ records: higher ${ }^{13} \mathrm{C}$ contents are recorded by all foraminiferal species during the glacial maxima of the late Pleistocene; they have been interpreted as indicative of minor stratification of the water masses and increased vertical convection (Vergnaud Grazzini et al., 1986; Oppo and Fairbanks, 1987). Thus, after $2.66 \mathrm{Ma}$, the response of the water-mass structures to global climatic changes became progressively similar to the modern one. The water budget may have changed from positive to negative, at least in the Tyrrhenian basin. This is supported by the fact that pollen diagrams indicate that the Tyrrhenian area was among the more humid ones within the Mediterranean, until about 2.4 Ma.

\section{The Pleistocene ${ }^{13} \mathrm{C}$ Record, from 1.66 $\mathrm{Ma}$ to the Present}

This part of the record corresponds to a long negative trend, with the lowest values of the whole record being reached near $0.52 \mathrm{Ma}$ for $G$. bulloides and near $0.7 \mathrm{Ma}$ for $G$. ruber. The Pleistocene starts with a marked negative $\delta^{13} \mathrm{C}$ oscillation between 1.65 and $1.50 \mathrm{Ma}$, which is more pronounced in the $G$. bulloides record (Figs. 8 and 9). A negative ${ }^{13} \mathrm{C}$ excursion is also recorded for the same time-interval at the Vrica section in southern Italy by G. bulloides (Fig. 10), at Hole 552A (benthic foraminifers; Shackleton and Hall, 1984), and at Site 548 (G. bulloides; Vergnaud Grazzini and Saliège, 1984) in the North Atlantic. In the early Pleistocene (Fig. 9) average $\delta^{13} \mathrm{C}$ values of $G$. bulloides start to decrease but they remain higher than modern ones during glacial episodes. The late Pleistocene carbon isotope signal of planktonic and benthic Mediterranean foraminifers bears strong regional overprints which are, in part, basin-dependent. As stated above, this results, for instance for the last glacial cycle, in opposite trends between the ${ }^{13} \mathrm{C}$ records of the open oceans (either Atlantic or Pacific) and those of the Mediterranean (Broecker,1982; Vergnaud Grazzini et al., 1986; Vergnaud Grazzini and Saliège, 1987; Glaçon and Vergnaud Grazzini, 1986; Capotondi et al., 1987). The ${ }^{13} \mathrm{C}$ content of species like $G$. ruber in the eastern Mediterranean basin or G. bulloides and Cibicides in the west Mediterranean and west of the Gibraltar sill, below the Mediterranean Outflow, is high during the glacial time and decreases during deglacial times. More generally, at least for the last $125 \mathrm{k} . \mathrm{y}$. in the Western Basin and the last 350 k.y. in the Eastern Basin, foraminiferal calcite records higher $\delta^{13} \mathrm{C}$ values during glacial times and lower $\delta^{13} \mathrm{C}$ values during deglacial times.
A detailed examination of the ${ }^{13} \mathrm{C}$ record of $G$. bulloides for the last million years (Fig. 9A) shows that $\delta^{13} \mathrm{C}$ values higher than modern one $(\sim 0.77 \%)$ are reached during isotopic stage 2 , part of stage 3 , substage 5.4 , substage 7.5 , stage 8 , the transition between stage 9 and stage 10, part of stage 10, "glacial" substages of stage 11 , stage 12 , and stage 18 . Most of these stages or substages correspond to glacial stages (even numbers). There are however some exceptions to the rule such as stage 6 and stage 16. Stage 6 in fact corresponds in the eastern Mediterranean to an episode of high stratification of the water masses which led to the stagnation of the basin and the deposition of sapropel (S6). This sapropel, however, has not been described in the Tyrrhenian basin.

\section{CONCLUSIONS}

The Pliocene-Pleistocene climatic evolution recorded at the Tyrrhenian Sites 653 and 654 is characterized by an overall increase in the average oxygen isotopic compositions of $\sim 1.7 \%$ for $G$. bulloides and $\sim 1.5 \%$ for $G$. ruber. The increase recorded by $G$. bulloides is higher by $0.85 \%$ o than that recorded at the same time, by the same species, in the Atlantic. In addition, since the early Pliocene, the amplitude of the $\delta^{18} \mathrm{O}$ fluctuations recorded in the Mediterranean was higher than in the Atlantic; in the Tyrrhenian basin, this amplitude increased in two steps: an earlier step around $0.95 \mathrm{Ma}$ and a second step around 0.34 Ma. Changes in the amplitude of the $\delta^{18} \mathrm{O}$ fluctuations have also been reported in the open ocean records, near 0.9 and $0.4 \mathrm{Ma}$, but the precise timing of these changes is still in debate. In the Mediterranean this resulted in late Pleistocene glacial-interglacial $\delta^{18} \mathrm{O}$ fluctuations with an amplitude twice that of the early Pliocene. Since the early Pliocene, therefore, the Mediterranean acted as an amplifier of the global isotopic signal. These data are related to a dryness increase and to the strong modifications of the water budget as the Mediterranean basin evolved toward a concentration basin. To a lesser degree they also indicate a cooling trend which is reflected by the changes in the faunal associations, in particular around $2.4 \mathrm{Ma}$ and near the Pliocene-Pleistocene boundary. The time interval between 3.1 and $2.2 \mathrm{Ma}$ corresponds to the transition between the preglacial and the glacial Pliocene. It is characterized by a progressive increase of the average $\delta^{18} \mathrm{O}$ values of $G$. obliquus while $G$. bulloides records more rapid and strong positive oscillations within a shorter period (2.67-2.46 Ma). The base of the Pleistocene is marked by a long term increase in the $\delta^{18} \mathrm{O}$ values of $G$. bulloides occurring between 1.55 and 1.3 Ma which is also recorded in the reference section of Vrica (southern Italy). It suggests a new step increase in dryness, resulting in a specific isotopic signal for the Mediterranean.

The $\delta^{13} \mathrm{C}$ data show that the Pliocene was a more productive period than the Pleistocene. An important decrease in the production of phytoplankton occurred around 1.7 Ma. At the same time (near $1.65 \mathrm{Ma}$ ) a strong negative ${ }^{13} \mathrm{C}$ oscillation which is recorded by $G$. bulloides at Hole $653 \mathrm{~A}$ as well as in the Vrica section, suggests a strengthening of the thermo-halocline with a depleted oxygen level at rather shallow water depth. Episodes of higher vertical mixing are recorded since the early Pliocene but are a dominant feature of the glacial intervals after $2.6 \mathrm{Ma}$. A paleoceanographic model, similar to that of the late Pleistocene glacial cycles, is suggested for the glacial cycles of the middle Pliocene.

All these data point to important climatic changes in the Tyrrhenian basin in the interval 3.1-2.2 Ma. The ${ }^{13} \mathrm{C}$ record is specific to the Mediterranean. This emphasizes the fact that changes in the $\mathrm{CO}_{2}$ balance within the different water masses as well as in the primary production did not follow the global trends of the open ocean. 
A

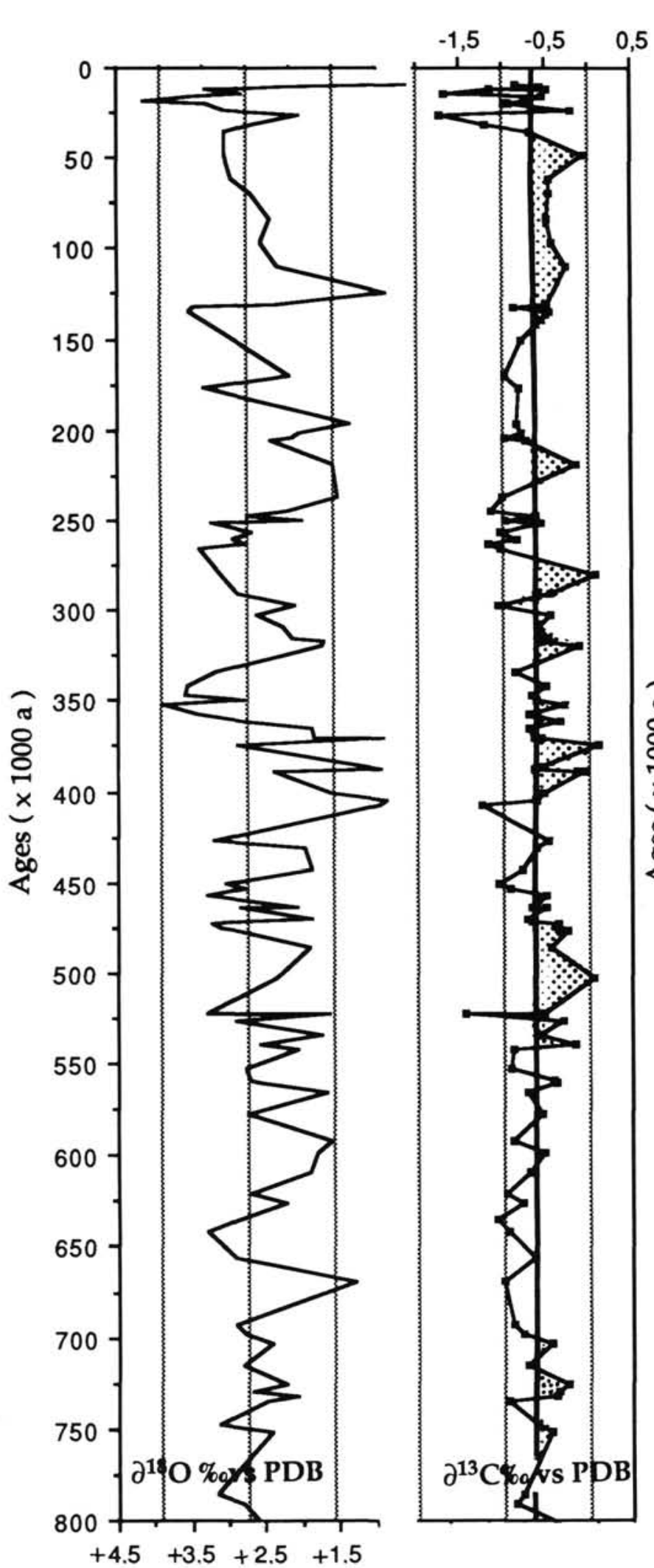

B $\quad 0.7-1.8 \mathrm{Ma}$

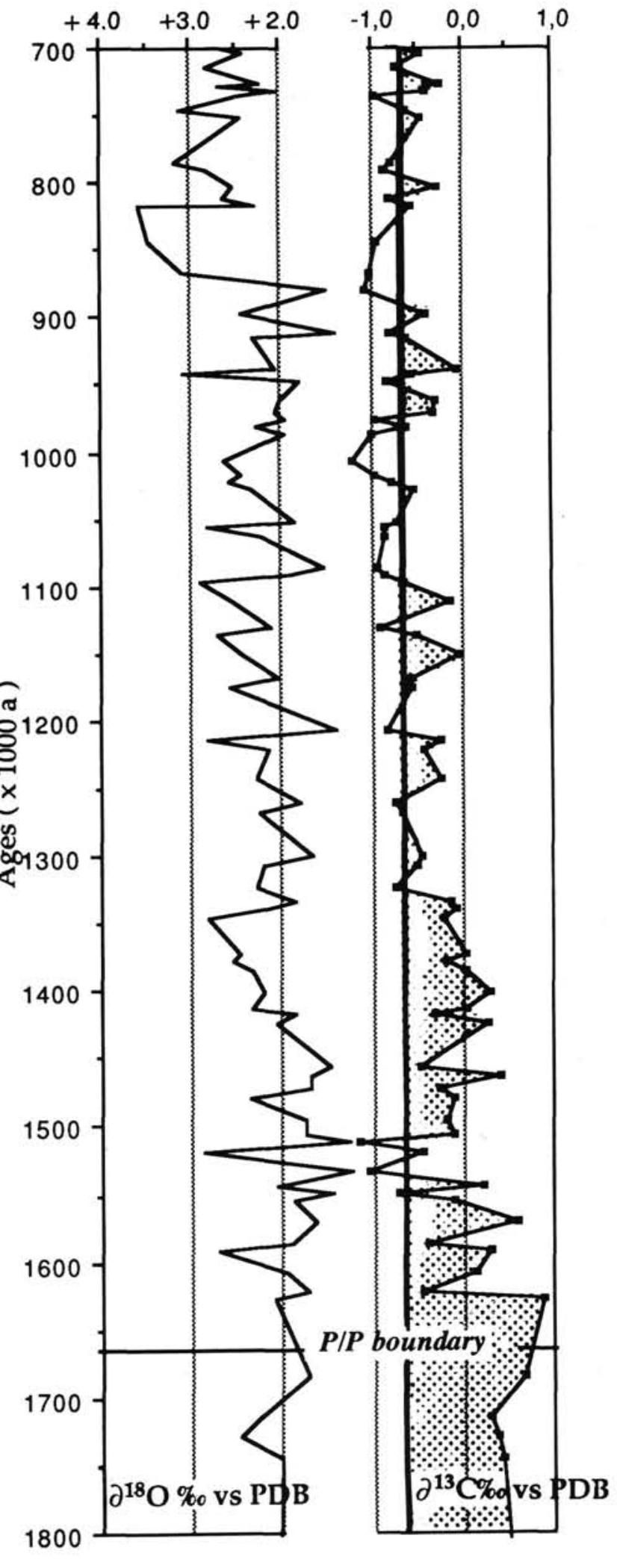

Figure 9. The Pleistocene $\delta^{13} \mathrm{C}$ record of $G$. bulloides at ODP Hole 653A reported against the time scale defined above and compared to the oxygen isotope stratigraphy. A. For the last $0.8 \mathrm{Ma}$. B. From the base of the Pleistocene to $0.8 \mathrm{Ma}$. Shadow areas indicate episodes with $\delta^{13} \mathrm{C}$ values higher than the modern average. 


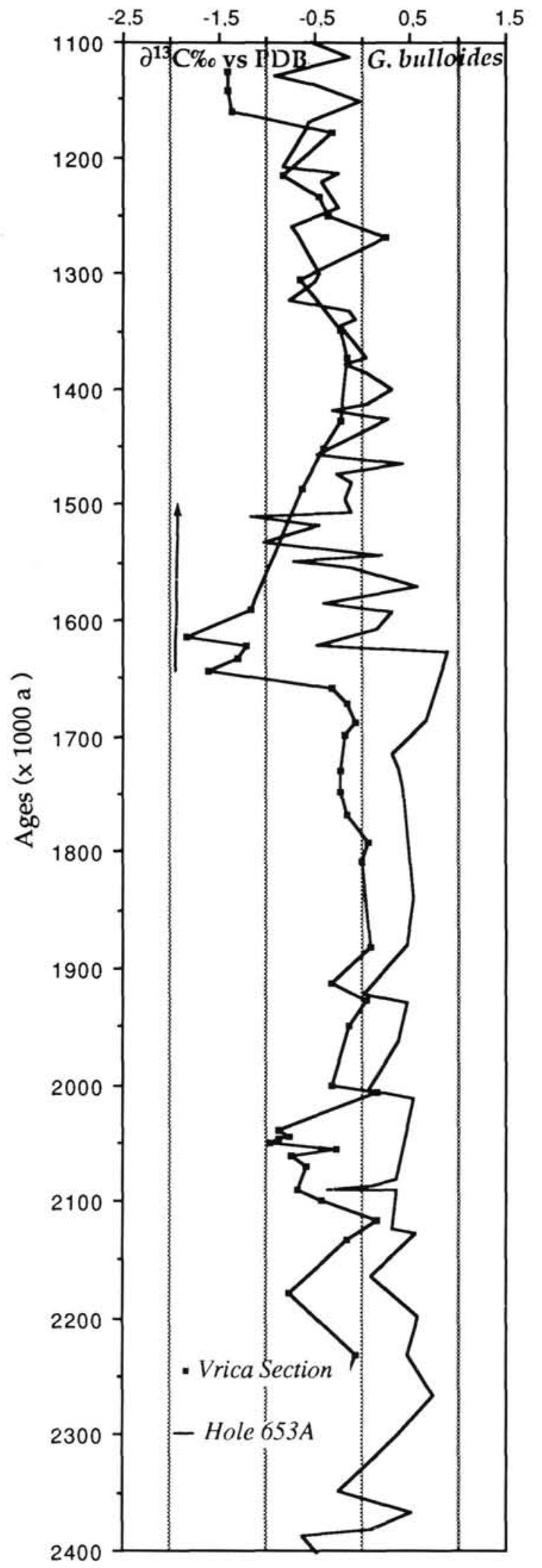

Figure 10. The ${ }^{13} \mathrm{C}$ records of $G$. bulloides at Hole $653 \mathrm{~A} \mathrm{(-)}$ and at the Vrica section (southern Italy) ( $\square$ ) for the period from 2.4 to $1.1 \mathrm{Ma}$.

\section{ACKNOWLEDGMENTS}

The authors acknowledge G. Glaçon for countless hours spent in selecting samples for stable isotope analyses as well as for her help in determination of foraminifer species, identification of major bio-events, and very constructive discussions. Thanks are extended to C. deVisser and $\mathrm{H}$. Chamley for having provided additional samples. We are grateful to two anonymous reviewers for their insightful review of the manuscript as well as to Dr. K. Kastens, J. Mascle, and F. McCoy for useful comments and suggestions to improve the manuscript. This is Contribution no. 40 to the C.N.R.S.-I.N.S.U.-D.B.T. Message sédimentaire Project. This study was partly funded through the ASP-ODP grant no. 981015 of the Centre National de la Recherche Scientifique and by the DBT Project of the Institut National des Sciences de l'Univers.

\section{REFERENCES}

Berger, A., in press. Paleo-insolation at the Plio-Pleistocene Boundary. In Evolution climatique du domaine méditerranéen au Néogène. RCMNS Interim Colloquium, Sept. 1987.

Benson, M. L., 1975. The origin of the psychrosphere as recorded in changes of deep sea Ostracode assemblages. Lethaia 8:69-83.

Berggren, W. A., Kent, D. V., and Van Couvering, J. A., 1985. Neogene geochronology and chronostratigraphy. In Snelling, N. J. [Ed.], The chronology of the Geological record. Lond. Geol. Soc. Mem, 10: 211-260.

Broecker, W. S., 1982. Ocean chemistry during glacial time. Geochim. Cosmochim. Acta 46:1689-1705.

Capotondi, L., Borsetti, A. M., and Vergnaud Grazzini, C. R., 1987. Biostratigraphie et composition isotopique de Foraminifères planctoniques des derniers 140000 ans dans la région de Minorque (Méditerranée occidentale). C.R. Acad. Sc. Paris, 305,11:493-498.

Combourieu Nebout, N., 1987. Les premiers cycles glaciaire-interglaciaire en région méditerranéenne d'après l'analyse palynologique de la série Plio-Pléistocène de Crotone (Italie). [Ph.D. dissert.]. Montpellier Univ.

Cravatte, J., and Suc, J. P., 1981. Climatic evolution of North Western Mediterranean area during Pliocene and early Pleistocene by pollen analysis and foraminifera of drill AUTAN 1. Chronostratigraphic correlations. Pollen and Spores, Paris, 23,2:247-258.

Duplessy, J. C., 1972. La géochimie des isotopes stables du carbone dans la mer. [Ph.D. dissert.] Univ. Paris.

Emiliani, C., 1966. Paleotemperature analysis of Caribbean Cores P. 6304-8 and P. 6304-9 and a generalized paleotemperature curve for the past 425,000 years. J. Geol., 74:103-104.

Fillon, R. H., and Williams, D. F., 1983. Glacial evolution of the PlioPleistocene: Role of continental and Arctic Ocean ice sheets. Palaeogeogr. Palaeoclimatol. Palaeoecol. 42:7-33.

Glaçon, G., and Vergnaud Grazzini, C. R., 1986. Les isotopes du carbone, outil stratigraphique pour les derniers 350000 ans en Méditerranée orientale. C.R. Acad. Sc., Paris, 303,18:1675-1678.

Gudjonsson, L., 1987. Local and global effects on the early Pliocene Mediterranean stable isotope records. Mar. Micropaleontol., 12: 241-253.

Gudjonsson, L., and Van der Zwaan, G. J., 1985. Anoxic events in the Pliocene Mediterranean: stable isotope evidence of run-off. Proc. $K$. Ned. Akad. Wet., B, 88,1:69-82.

Hodell, D. A., Kennett, J. P., and Leonard, K. A., 1983. Climaticallyinduced changes in vertical water mass structure of the Vema Channel during the Pliocene: evidence from DSDP Sites 516A, 517, and 518. In Barker, P. F., Carlson, R. L., and Johnson, D. A., et al., lnit. Repts. DSDP, 72: Washington (U.S. Govt. Printing Office), 907-919.

Hodell, D. A., Williams, D. F., and Kennett, J. P., 1985. Late Pliocene reorganization of deep vertical water mass structure in the western south Atlantic: Faunal and isotopic evidence. Geol. Soc. Am. Bull., 96:495-503.

Imbrie, J., Hays, J. D., Martinson, D. G., Mclntyre, A., Mix, A. D., Morley, J. J., Pisias, N. G., Prell, W. L., and ShackIeton, N. J., 1984. The orbital theory of Pleistocene climate: support from a revised chronology of the marine $\delta^{18} \mathrm{O}$ record. In Berger, A., et al. [Eds.] Miankovitch and Climate, Dordrecht (D. Riedel), 269-305.

Jansen, E., and Sejrup, H. P., 1987. Stable isotope stratigraphy and amino-acid epimerization for the last 2.4 M.Y. at Site 610, Holes 610 and 610A. In Ruddiman, W. F., Kidd, R. B., Thomas, E., et al., 
Init. Repts. DSDP, 94: Washington (U.S. Govt. Printing Office), 879-887.

Jansen, J. H., Kuijpers, A., and Troestra, S. R., 1986. A Mid-Brunhes Climatic Event: Long-Term Changes in Global Atmosphere and Ocean Circulation. Science, 132:619-622.

Kastens, K. A., Mascle, J., et al., 1987. Proc. ODP, Init. Repts. 107: College Station, TX (Ocean Drilling Program).

Keigwin, L. D., 1987. Pliocene stable isotope record of Deep Sea Drilling Project Site 606: sequential events of ${ }^{18} \mathrm{O}$ enrichment beginning at 3.1 Ma. In Ruddiman W. F., Kidd, R. B., Thomas, E., et al., Init. Repts. DSDP, 94, Washington (U.S. Govt Printing Office), 911-920.

Keigwin, L. D., and Thunell, R. C., 1979. Middle Pliocene climatic change in the western Mediterranean from faunal and oxygen isotopic trends. Nature, 282:294-296.

Lacombe, H., Gascard, J. C., Gonella, J., and Bethoux, J. P., 1981. Response of the Mediterranean to the water and energy fluxes across its surface, on seasonal and interannual scales. Oceanol. Acta, 4:247255.

Leonard, K. A., Williams, D. F., and Thunell, R. C., 1983. Pliocene paleoclimatic and palaeoceanographic history of the South Atlantic Ocean: stable isotopic records from Leg 72 Deep Sea Drilling Project Holes 516A and 517. In Barker, P. F., Carlson, R. L., Johnson, D. A., et al., Init. Repts. DSDP, 72, Washington (U.S. Govt. Printing Office), 895-906.

Lona, F., 1962. Prime analisi paleontologiche nei depositi terziari, quaternari di Castell'Arquato: reperti di vegetazione da clima freddo sotto le formazioni calcaree ad Amphistegina. Boll. Soc. Geol. It., 81:89-91.

Loubere, P., 1988. Gradual Late Pliocene onset of glaciation: a deepsea record from the Northeast Atlantic. Palaeogeogr. Palaeoclimatol. Palaeoecol., 63:327-334.

Martinson, D. G., Pisias, N. G., Hays, J. D., Imbrie, J., Moore, T. C., Jr., and Shackleton, N. J., 1987. Age dating and the orbital theory of the ice ages: development of a high-resolution 0 to 300,000-year chronostratigraphy. Quatern. Res., 27:1-29.

Oppo, D. W., and Fairbanks, R. G., 1987. Variability in the deep and intermediate water circulation of the Atlantic Ocean during the past 25,000 years: Northern Hemisphere modulation of the Southern Ocean. Earth Planet. Sci. Lett., 86:1-15.

Paterne, M., Guichard, F., Labeyrie, J., Gillot, P. Y., and Duplessy, J. C., 1986. Tyrrhenian sea tephrochronology of the oxygen isotope record for the past 60,000 years. Mar. Geol., 72:259-285.

Pierre, C., Vergnaud Grazzini, C. R., Thouron, D., and Saliège, J. F., 1986. Compositions isotopiques de l'oxygène et du carbone des masses d'eau en Méditerranrée. Mem. Soc. Geol. It., 36:165-174.

Pons, A., Suc, J. P., Reille, M., and Combourieu Nebout, N., in press. The past history of dryness in regions with a mediterranean climate. In Di Castri, G., and Mooney, H. A. [Eds.] Time scales of water stress response of Mediterranean biota, Berlin.

Prell, W. L., 1982. Oxygen and carbon isotope stratigraphy for the Quaternary of Hole 502B: evidence for two modes of isotopic variability. In Prell, W. L., Gardner, J. V., et al., Init. Repts. DSDP, 68: Washington (U.S. Govt. Printing Office), 455-464.

1984. Covariance patterns of foraminiferal $\delta^{18} \mathrm{O}$ : an evaluation of Pliocene ice volume changes near 3.2 million years ago. Science, 226:692-694.

1985. Pliocene stable isotope and carbonate stratigraphy (Hole $572 \mathrm{C}$ and 573A): palaeoceanographic data bearing on the question of Pliocene glaciation. In Mayer, L., Theyer, L., et al.. Init. Repts. DSDP, 85: Washington (U.S. Govt. Printing Office), 723-734.

Raffi, S., 1986. The significance of marine boreal molluscs in the Early Pleistocene faunas of the Mediterranean area. Palaeogeogr., Palaeoclimatol. Palaeoecol., 52:267-289.

Rio, D., Sprovieri, R., and Raffi, I., 1984. Calcareous plankton biostratigraphy and biochronology of the Pliocene-Lower Pleistocene succession of the Capo-Rossello area (Sicily). Mar. Micropaleontol., 9:135-180.

Ruddiman, W. F., Raymo, M., and McIntyre, A., 1986. Matuyama 41.000-year cycles: North Atlantic Ocean and northern hemisphere ice sheets. Earth Planet. Sci. Lett., 80:117-129.

Ryan, W.B.F., 1972. Stratigraphy of the Late Quaternary sediments in the Eastern Mediterranean. In Stanley, D. J. (Ed.), The Mediterranean sea, Stroudsburg, PA (Dowden, Hutchinson and Ross), 149169.
Shackleton, N. J., Backman, J., Zimmerman, H., Kent, D. V., Hall, M. A., Roberts, D. G., Schnitker, D., Baldauf, J. G., Desprairies, A., Homrighausen, R., Huddlestun, P., Keene. J. B., Kaltenbach, A. J., Krumsiek, K. A. O., Morton, A. C., Murray, J. W., and Westberg-Smith, J., 1984. Oxygen isotope calibration of the onset of icerafting and history of glaciation in the North Atlantic region. $\mathrm{Na}$ ture, 307:620-623.

Shackleton, N. J., and Cita, M. B., 1979. Oxygen and carbon isotope stratigraphy of benthic foraminifers at Site 397: detailed history of climatic changes during the late Neogene. In von Rad, U., Ryan, W.B.F., et al., Init. Repts DSDP, 47A. Washington (U.S. Govt. Printing Office), 433-445.

Shackleton, N. J., and Hall, M. A., 1983. Stable isotope record of Hole 504 sediments: High resolution record of the Pleistocene. In Cann, J. R., Langseth, M. G., Honnorez, J., Von Herzen, R. P., White, S. W. et al. Init. Repts. DSDP, 69: Washington (U.S. Govt. Printing Office), 431-441.

1984. Oxygen and carbon isotope stratigraphy of Deep Sea Drilling Project Hole 552A: Plio-Pleistocene glacial history. In Schnitker, D. G. et al. Init. Repts DSDP, 81, Washington: (U.S. Govt. Printing Office), 599-609.

Shackleton, N. J., and Opdyke, N. D., 1976. Oxygen isotope and paleomagnetic stratigraphy of pacific core V 28-239, late Pliocene to latest Pleistocene. Geol. Soc. Am. Mem., 145:449-464.

1977. Oxygen isotope and paleomagnetic evidence for early Northern Hemisphere glaciation. Nature, 270:216-219.

Sprovieri, R., 1978. I foraminiferi bentonici della sezione Plio-Pleistocenica di Capo-Rossello (Agrigento,Sicily). Boll. Soc. Paleontol. It., $17: 68-97$.

Suc, J. P., 1984. Origin and evolution of the Mediterranean vegetation and climate in Europe. Nature, 307:429-432.

1986. Flores néogènes de Méditerranée occidentale. Climat et paléogéographie. Bull. Centres Rech. Explor. Prod. Elf-Aquitaine, $10: 477-488$

Suc, J. P., and Zagwijn, W. H., 1983. Plio-Pleistocene correlations between the northwestern Mediterranean region and northwestern Europe according to recent biostratigraphic and palaeoclimatic data. Boreas, 12:153-166.

Thunell, R. C., Williams, D. F., Raffi, I., Rio, D., and Sprovieri, R., 1985. Pliocene-Pleistocene history of the Mediterranean: results from deep sea and land-based marine records. Terra Cognita, 5:89.

Thunell, R. C., and Williams, D. F., 1983. The stepwise development of Pliocene-Pleistocene paleoclimatic and paleoceanographic conditions in the Mediterranean: oxygen isotopic studies of DSDP sites 125 and 132. Utrecht Micropaleontol. Bull., 30:11-127.

Thunell, R. C., Williams, D. F., and Howell, M., 1987. Atlantic-Mediterranean water exchanges during the late Neogene. Paleoceanography. 2:661-678.

Van der Zwaan, G. J., 1983. Quantitative analyses and the reconstruction of benthic foraminiferal communities. Utrecht Micropaleontol. Bull., 30:49-69.

Van der Zwaan, G. J., and Gudjonsson, L., 1986. Middle Miocene-Pliocene stable isotope stratigraphy and paleoceanography of the Mediterranean. Mar. Micropaleontol., 10:71-90.

Van Harten, D., 1984. A model of estuarine circulation in the Pliocene Mediterranean based on new ostracod evidence. Nature, 312:359361.

Vergnaud Grazzini, C. R., 1973. Etude écologique et isotopique de foraminifères actuels et fossiles de Méditerranée. [Ph.D. dissert.]. Univ. Paris.

1975. ${ }^{18} \mathrm{O}$ changes in foraminiferal carbonates during the last $10^{5}$ years in the Mediterranean Sea. Science, 190:272-274.

1983. Reconstruction of Mediterranean Late Cenozoic hydrography by means of carbon isotope analyses. Utrecht Micropaleontol. Bull., 30:25-47.

1985. Mediterranean Late Cenozoic stable isotope record: stratigraphic and paleoclimatic implications. In Stanley, D. J., and Wezel, F. C. [Eds.], Geological Evolution of the Mediterranean Ba$\sin$. New York (Springer), 413-451.

Vergnaud Grazzini, C. R., Devaux, M., and Znaidi, J., 1986. Stable isotope "anomalies" in Mediterranean Pleistocene records. Mar. Micropaleontol,. 10:35-69.

Vergnaud Grazzini, C. R., Ryan, W.B.F., and Cita, M.B., 1977. Stable isotopic fractionation, climate change and episodic stagnation in the 
eastern Mediterranean during the Late Quaternary. Mar. Micropaleontol., 2:353-370.

Vergnaud Grazzini, C. R., Grably, M., Pujol, C., and Duprat, J., 1983. Oxygen isotope stratigraphy and paleoclimatology of southwestern Atlantic Quaternary sediments (Rio Grande Rise) at Deep Sea Drilling Project Site 517. In Barker, P. F., Carlson, R. L., Johnson, D. A., et al., Init. Repts DSDP, 72: Washington (U.S. Govt. Printing Office), 871-884.

Vergnaud Grazzini, C. R., Van der Zwaan, G. J., Iaccarino, S., and Gudjonsson. L., 1985. Mediterranean climate and hydrology since the late Miocene: Oxygen and carbon isotope records. VIIIth RCMNS Congress. Budapest. Abstr.

Vergnaud Grazzini, C. R., and Saliège, J. F., 1984. Pleistocene climatic changes in surface waters in the Northeastern Atlantic: oxygen and carbon isotopic compositions of planktonic foraminifers at Deep Sea Drilling Project sites 548 and 549. In Graciansky, P. C., de, Poag, C. W., et al., Init. Repts DSDP, 80, Washington, (U.S. Govt. Printing Office), 793-803.

1987. Carbon-13 depletion in Mediterranean Foraminifera during the last deglaciation. Terra Cognita, 7:214.
Williams, D. F., Thunell, R. C., Tappa, E., Rio, D., and Raffi, I., 1988. Chronology of the Pleistocene oxygen isotope records: $0-1.88$ m.y. B.P.. Palaeoeogr., Palaeoclimatol. Palaeoecol., 64:221-240.

Zachariasse, W. J., and Spaak, P., 1983. Middle Miocene to Pliocene paleoenvironmental reconstructions of the Mediterranean and adjacent Atlantic ocean: planktonic foraminiferal records of Southern Italy. Utrecht Micropaleontol. Bull., 30:91-110.

Zagwin, W. H., 1974. The Plio-Pleistocene boundary in western and southern Europe. Boreas, 3:75-97.

Zimmermann, H. B., Shackleton, N. J., Backman, J., Kent, D. V., Baldauf, J. G., Kaltenback, A. J., and Morton, A. C., 1985. History of Plio-Pleistocene Climate in the Northeastern Atlantic Deep Sea drilling Project Hole 552A. In Roberts, D. G., Schnitker, D., et al., Init. Repts DSDP, 81: Washington (U.S. Govt. Printing Office), 861-876.

Date of initial receipt: 12 January 1988

Date of acceptance: 17 January 1989

Ms 107B-153 